\title{
A case study in connectomics: the history, mapping, and connectivity of the claustrum
}

\section{Carinna M. Torgerson and John D. Van Horn*}

Department of Neurology, Laboratory of Neuro Imaging, Institute of Neuroimaging and Informatics, University of Southern California, Los Angeles, CA, USA

\section{Edited by:}

Mihail Bota, University of Southern

California, USA

\section{Reviewed by:}

Graham J. Galloway, The University of Queensland, Australia

David Reser, Monash University,

Australia

\section{*Correspondence}

John D. Van Horn, Laboratory of Neuro Imaging, The Institute for Neuroimaging and Informatics, Keck School of Medicine, University of Southern California, 2001 North

Soto Street - Room 102, Los

Angeles, CA 90032, USA

e-mail: jvanhorn@usc.edu
The claustrum seems to have been waiting for the science of connectomics. Due to its tiny size, the structure has remained remarkably difficult to study until modern technological and mathematical advancements like graph theory, connectomics, diffusion tensor imaging, HARDI, and excitotoxic lesioning. That does not mean, however, that early methods allowed researchers to assess micro-connectomics. In fact, the claustrum is such an enigma that the only things known for certain about it are its histology, and that it is extraordinarily well connected. In this literature review, we provide background details on the claustrum and the history of its study in the human and in other animal species. By providing an explanation of the neuroimaging and histology methods have been undertaken to study the claustrum thus far-and the conclusions these studies have drawn-we illustrate this example of how the shift from micro-connectomics to macro-connectomics advances the field of neuroscience and improves our capacity to understand the brain.

Keywords: claustrum, connectomics, macro-scale, micro-scale, Wilson's Disease, consciousness

\section{INTRODUCTION}

Macro-scale connectomics, the study of neuronal connections between two or more regions of the brain, combines the principles of functional specialization and functional integration. While there are only a mere $30,000-40,000$ protein-encoding human genes, and nearly 1.5 million single nucleotide polymorphisms (SNPs), there may be an astonishing $10^{15}$ neuronal connections in the human brain (Lander et al., 2001; Sporns et al., 2005). Despite such complexity, the use of modern neuroimaging is ushering in a new vision for describing the wiring of the brain. While graph theory, diffusion imaging, and even functional imaging are still in their relative infancy, researchers have never possessed more appropriate tools for decoding the enigmas of the brain. Connectomics analysis is particularly revelatory for small, highly connected structures, like the claustrum.

Indeed, the claustrum serves as an informative case study for examining the boon that connectomics research has brought to the field of neuroscience; it has remained one of the most mysterious structures in the brain since the 17th century. Its name, in fact, means "hidden away" (Crick and Koch, 2005). Now, macro-scale connectomics allow for new analysis that may help unlock the secrets of the enigmatic structure. In this literature review, we discuss the limitations that have led to the claustrum to be investigated through a connectomics lens in the human and in other animal species, even before Sporns, Tononi, Hagmann, Bullmore, and others launched the modern connectomics movement. Our review includes descriptions of neuroimaging and histology studies tracing claustral connectivity, its putative role(s) in various neural systems, its reported influence in neurological syndromes, and examines the recent flurry of interest in the macro-connectomics of the claustrum.

\section{BACKGROUND}

The structure of the claustrum is visible as early as 1672 in the drawings of Thomas Willis (Bayer and Altman, 1991) who first proposed that higher cognitive functions arose from the convolutions of the cerebral cortex, rather than the ventricles (Molnár, 2004). Karl Friedrich Burdach, first described the claustrum (using the German word "vormauer"), however, in his seminal work Von Baue und Leben des Gehirns, in the early 19th century (Parent, 2012). Burdach himself credited the first illustrated depiction (Figure 1) of the structure to the 1786 drawings by Marie-Antoinette's personal physician, Félix Vicq-d'Azyr, who not only discovered the substantia nigra, but also provided the most detailed drawings of the basal ganglia of his time (Parent, 2012). Perhaps the first person to appreciate how crucial the claustrum is in multi-modal processing was Theodor Meynert, the director of the psychiatric clinic at the University of Vienna in the late 19th century. Investigating aphasia, Meynert noted that many post-mortem examinations of aphasic patients turned up pathological changes between the insula and the Sylvian fissure. The general belief at the time held that the entire cortex surrounding the Sylvian fissure was dedicated to speech. Meynert hypothesized that the claustrum contained an "acoustic field" that corresponded to the beginning of the Acousticusstrang, or "acoustic tract" (Eling, 1994). Information from the acoustic nerve, he posited, was associated with the speech system through spindle-shaped association cells in the claustrum, before being transmitted to the Sylvian fissure. His evidence for this relationship was the well-understood relationship between the claustrum and other "association systems" in the brain (Eling, 1994).

Classification of the claustrum is not a straightforward process. It cannot be accurately described as strictly cortical or subcortical, 


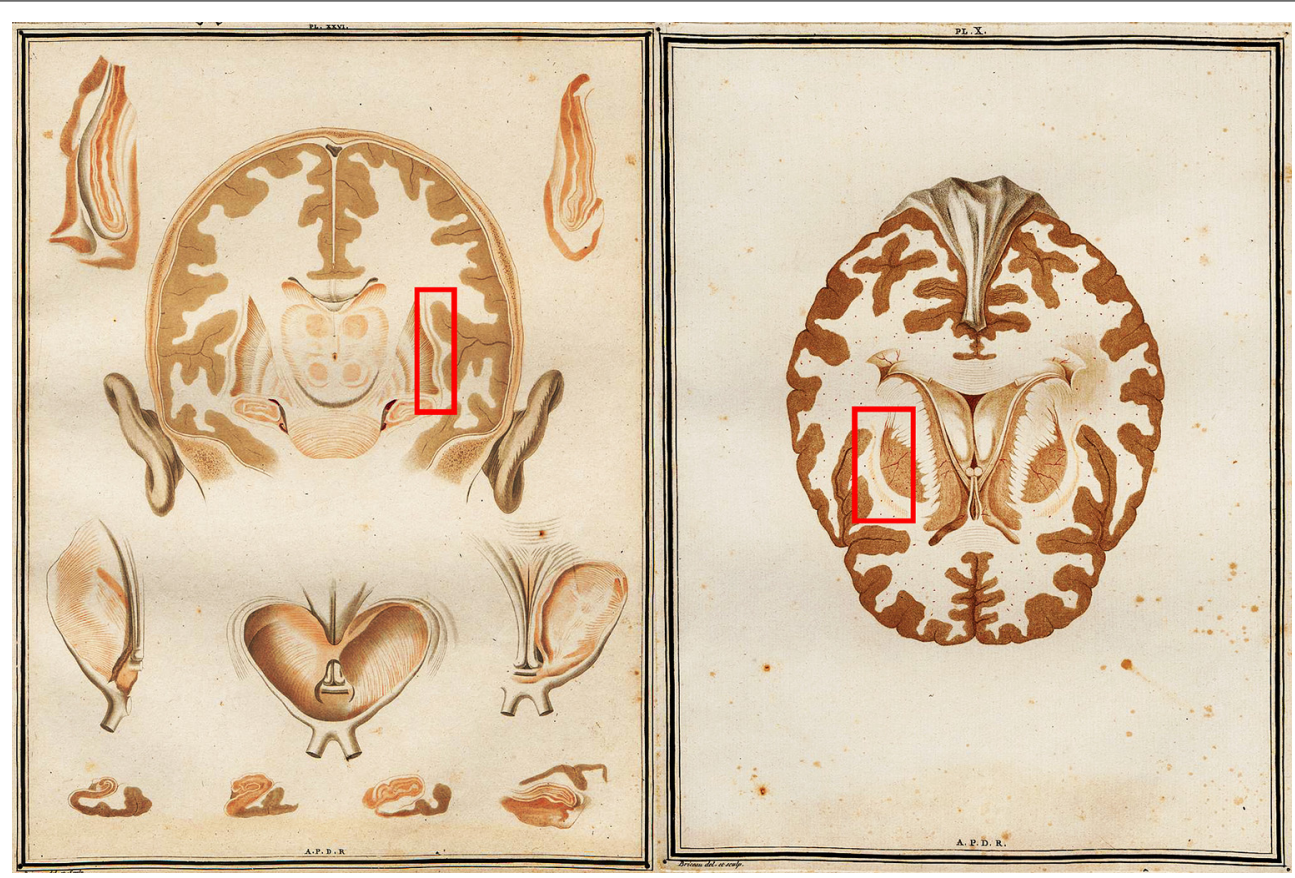

FIGURE 1 | Two early views of the brain which feature the human claustrum from Vicq D'Azyr's Traité d'anatomie et de physiologie. Red boxes applied by the authors to indicate the location of the left and right claustra.

as it possesses the laminar organization and characteristic pyramidal somata of cortical regions in Insectivora (Narkiewicz and Mamos, 1990), but also contains some notably subcortical cell types (Mathur et al., 2009). Although prominent researchers such as Brodmann and Wernicke suggested that the claustrum represents the innermost layer of the insula due to its close proximity (Landau, 1919) it is somewhat unsatisfactorily included as part of the basal ganglia today. As a case in point, a search for the claustrum on PubMed will automatically also search for the broader term "basal ganglia," presumed to reflect the fact that claustral afferent inputs are believed to be similar to the striatum, although its efferents connect directly to the cortex without passing through a thalamic relay (Salerno et al., 1984). These efferent connections have a relatively slow conduction speed, which suggests they are small in diameter and/or poorly myelinated. More recently, Pirone et al. (Pirone et al., 2012) have concluded that the more likely ontological relationship of the human claustrum is with insula, rather than basal ganglia, based on immunostaining of human tissue. The detailed overview provided by Druga (2014) indicates a pallial origin of the claustrum, whereas the striatum appears to be of sub-pallial origin. Puelles (2014) notes that various techniques throughout history have suggested the claustrum could be derived from insular cortical strata, the subpalium/basal ganglia, non-insular pallium, or even that different claustral subdivisions arose from distinct regions. He does, however, note that recent genoarchitectonic and immunocytochemical investigations seem to have reinstated the insular derivative theory. Not only do these new investigations concur with the claustral differences noted in species with and without extreme capsules, they also bolster the recent discussions of the role of the claustrum in consciousness (see Methods for Studying the Claustrum, below), since the insula itself has been recently implicated as a neural site for conscious awareness (Craig, 2009).

Despite its considerable-and controversial-history, the structure itself is diminutive (Figure 2A). As represented in the Talairach and Tournoux (1988) atlas, the claustrum is located medial to the insular cortex and lateral to the putamen from between -4 and $+16 \mathrm{~mm}$ relative to the AC-PC plane. The right claustrum has an approximate average surface area of $1551.15 \mathrm{~mm}^{2}$ and volume of $828.83 \mathrm{~mm}^{3}$ while the left claustrum has a surface area of $1439.16 \mathrm{~mm}^{2}$ and volume of $705.82 \mathrm{~mm}^{3}$ (Kapakin, 2011). The noticeable asymmetry (Figure 2B) in structure, volume, and average anisotropy (Cao et al., 2003) may relate to function, as the right claustrum, but not the left, has been shown to react differently to congruent and incongruent stimuli (Naghavi et al., 2007). Although the fine-grained anatomy of the claustrum in the human remains poorly understood, it is reasonable to expect that there exist major divisions present as in all of the species examined to date, i.e., a ventral, "endopiriform" and a dorsal "insular" component. Given a putative shared history with the insula, neural sub-divisions may be present mirroring, in part, those of adjacent insular cortex (Puelles, 2014). Thus, the claustrum is unlikely to function as a single uniform body, per se, but have finely interconnected sub-divisions. Lastly, it remains unclear whether blood arrives through the vessels penetrating the insula (Edelstein and Denaro, 2004) or from the deep and superficial sections of the middle cerebral artery (Crick and Koch, 2005).

Embryonic observation shows that neurons settle in the claustrum 4-6 days after the peak of neurogenesis (Bayer and Altman, 


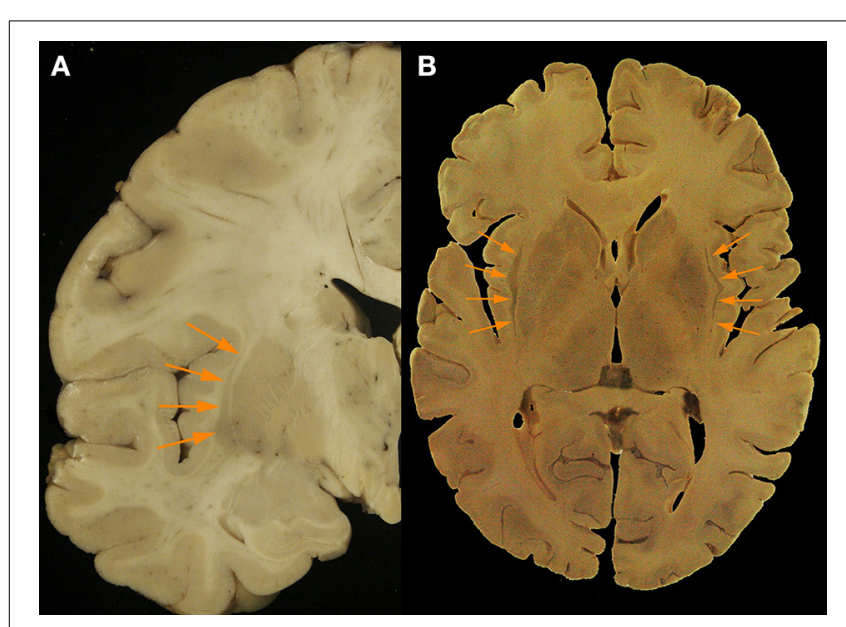

FIGURE 2 | (A) Axial post mortem anatomical view of the claustrum (orange arrows; taken from the Rasmussen Neuroanatomical Collection housed at the UCLA David Geffen School of Medicine, Los Angeles, CA), and $\mathbf{( B )}$ a separate coronal view of the claustral body in the left hemisphere from post-mortem tissue (orange arrows). Modern neuroimaging computational segmentation algorithms do not consider the claustrum due to the particular difficulty of extracting it from the surrounding tissues.

1991). As most structures do with age, the claustrum increases in volume through middle age and decreases again in old age (Wisco et al., 2008). It shares its ontogeny with the insular cortex, but not with another of its neighbors, the putamen (Pirone et al., 2012). The claustrum is very closely related to both the insula and external capsule. In fact, removal of the white fibers in the external capsule leads to the dorsal claustrum is also being removed. Removing all the fibers from the external capsule that merge in the dorsal claustrum leads to removal of the dorsal claustrum, leaving the putamen exposed, without a lateral covering (Fernandez-Miranda et al., 2008b). The dorsal external capsule is mainly composed of projection (intra-hemispheric corticosubcortical) and not association (intra-hemispheric inter-lobar cortico-cortical) fibers (Fernandez-Miranda et al., 2008a).

\section{METHODS FOR STUDYING THE CLAUSTRUM}

The claustrum has been the subject of a relatively small body of research, although the pace of research is increasing in today's era of diffusion imaging and macro-connectomics (Table 1). In fact, many studies that mention the claustrum only find its involvement to be a result of their research question, but do not intend to focus on the structure, nor define it particularly carefully. Until recently, the literature was predominantly composed of animal studies (Table 2). Human in vivo claustrum studies seem to be a phenomenon almost exclusive to the 21st century. While the paucity of neuroimaging technology throughout most of history can account in some part for the scarceness of claustral studies, high-resolution imaging of other small, non-cortical structures was undertaken far earlier. The majority of claustrum investigations have examined the microscopic detail of the claustrum: its neuronal composition (Sherk and LeVay, 1981; Bayer and Altman, 1991; Mathur et al., 2009; Smythies et al., 2012b); its afferents to a single cortical region (Edelstein and Denaro, 2004;
Smith et al., 2012); and its excitatory or inhibitory properties (Sherk and LeVay, 1981; Shima et al., 1996). Only a relative few at the time of this writing have attempted to create a macro-scale picture of how the claustrum fits within a larger context-some more recent studies attempt to discover its role in cortico-cortical networks (Hadjikhani and Roland, 1998; Tanne-Gariepy et al., 2002; Poeppl et al., 2014) or to understand its function by analyzing how it alters the electrical frequency of incoming signals (Smythies et al., 2014).

Some unique characteristics have held back research of the claustrum. It is important to note that imaging of the structure is infamously challenging. Early neuroscientists often examined the brains of the deceased in order to relate abnormalities with some functional deficit the patient experienced during life. The claustrum cannot be studied in this way, as researchers have yet to induce a lesion affecting the claustrum without also affecting neighboring structures, although some natural lesions have been reported in epileptic subjects (Sperner et al., 1996; Duffau et al., 2007). Even with the advent of histological staining techniques, it is incredibly difficult to localize an injection in the claustrum without some tracer spreading. The emergence of neuroimaging could not address this limitation of size, as fine-scale structures such as the tiny claustrum can be severely distorted by high-resolution MRI (Konukoglu et al., 2013). Conversely, the claustrum is simply not visible in some low-resolution MR imaging; Meng et al. note that in the developing brain the claustrum is not visible even at 11.7-T MRI, but can be seen on T2-weighted images at 7T (Meng et al., 2012). The difficulty of capturing the claustrum has in fact helped researchers compute signal-to-noise and contrast-to-noise ratios for image restoration because it can only be seen under specific conditions (Konukoglu et al., 2013). In terms of functional imaging, temporal resolution is already notoriously poor. It is not unusual for fMRI spatial resolution to be larger than the width of the claustrum, and therefore attributing any function to just the claustrum runs the risk of ascribing tasks actually carried out in the insula or external capsule to their claustral neighbor. Such misattribution is particularly dangerous in light of the suggestions that the claustrum and insula connect to very different regions (Park et al., 2012).

Within the last 5 years, tractography studies of the claustrum have been undertaken in hopes of obtaining a broader picture of how the claustrum relates to the rest of the brain. An example rendering based upon diffusion imaging of the claustrum is shown in Figures 3A-D. Researchers have begun to examine how the claustrum connects functionally disparate cortical networks, and to attempt to extrapolate from these individual examples a larger idea of the role the claustrum plays in the brain. Presumably, the logic of this is that the claustrum performs the same function for all networks in which it participates. The strategy for assessing function seems to be to analyze networks and structures that we understand well, and attribute any unexplainable interaction to the claustrum. This process creates a reactionary chain of research in which one aspect of claustral function is asserted, and then argued against with evidence from a different claustrocortical network. For example, the reciprocal connections from the visual cortex to the claustrum have been used to suggest segregation of function in the claustrum, and yet, analyses of 


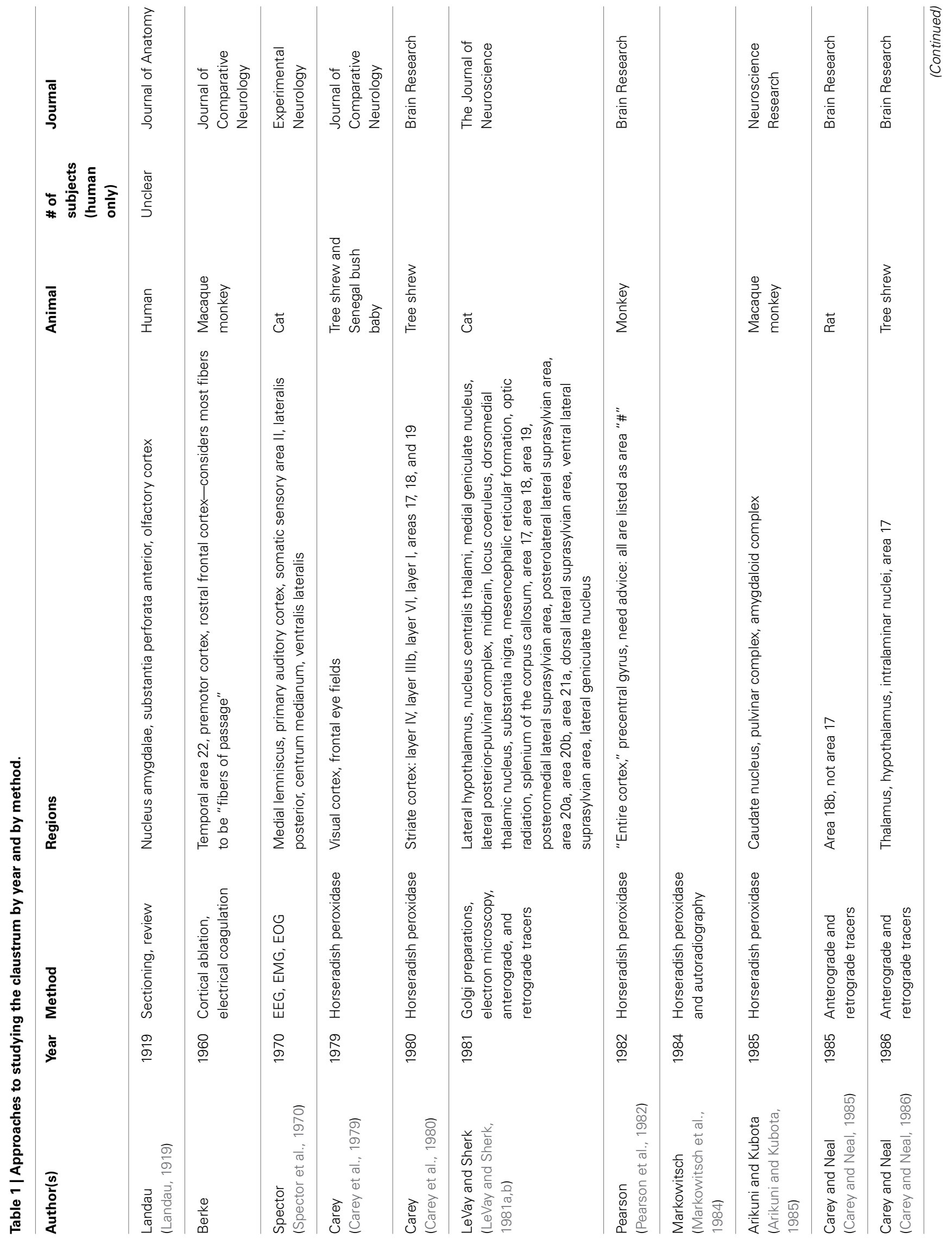




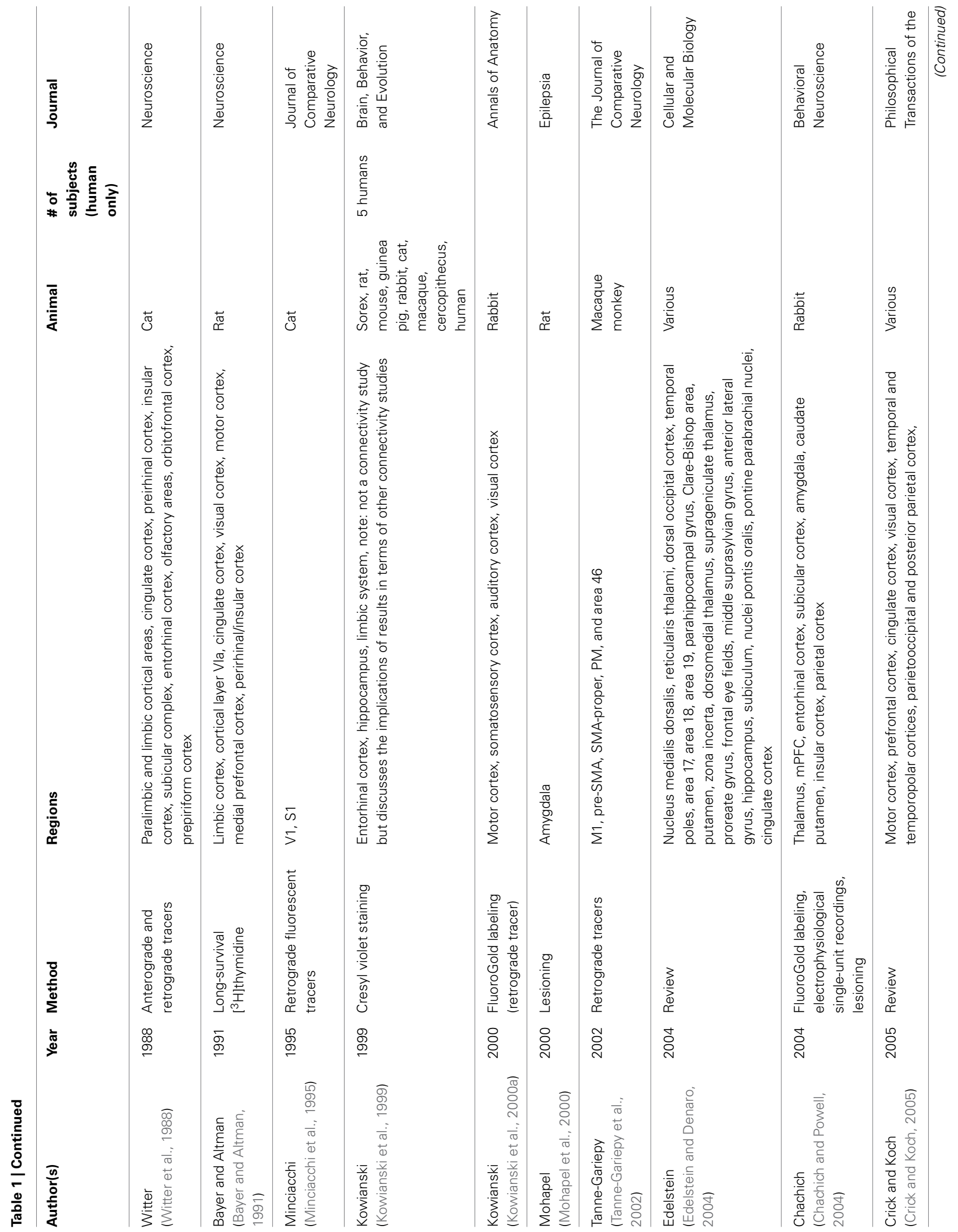




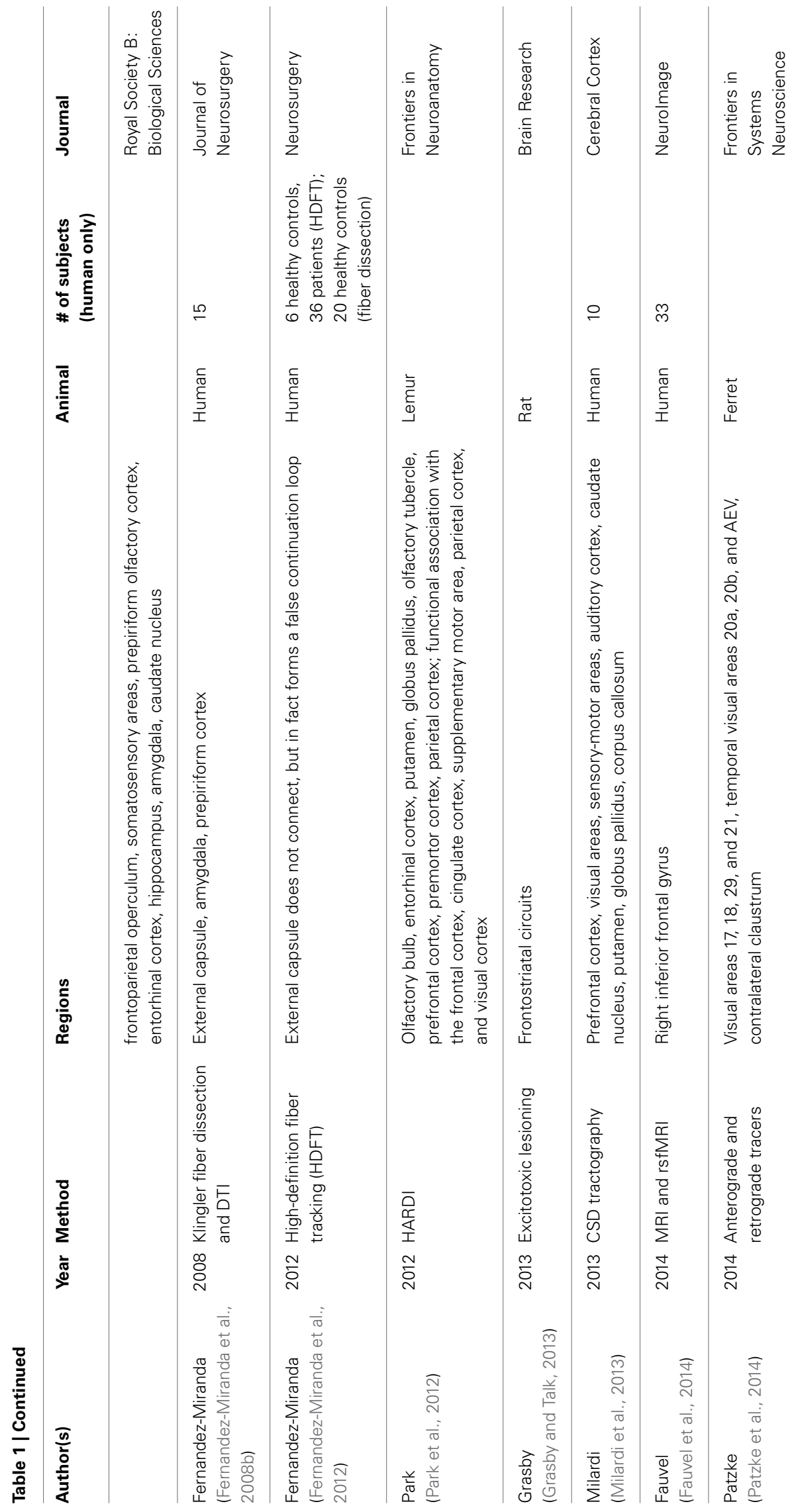




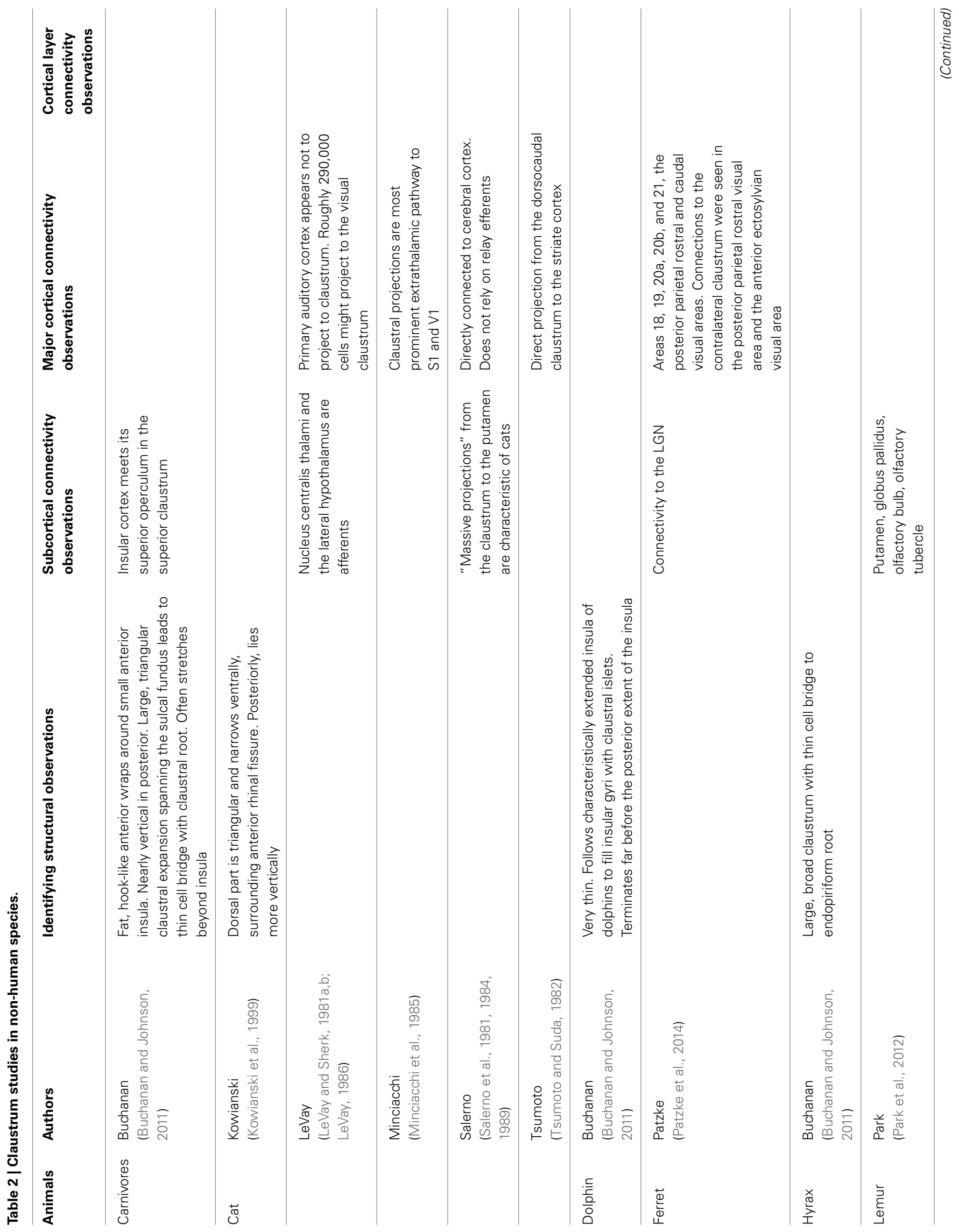




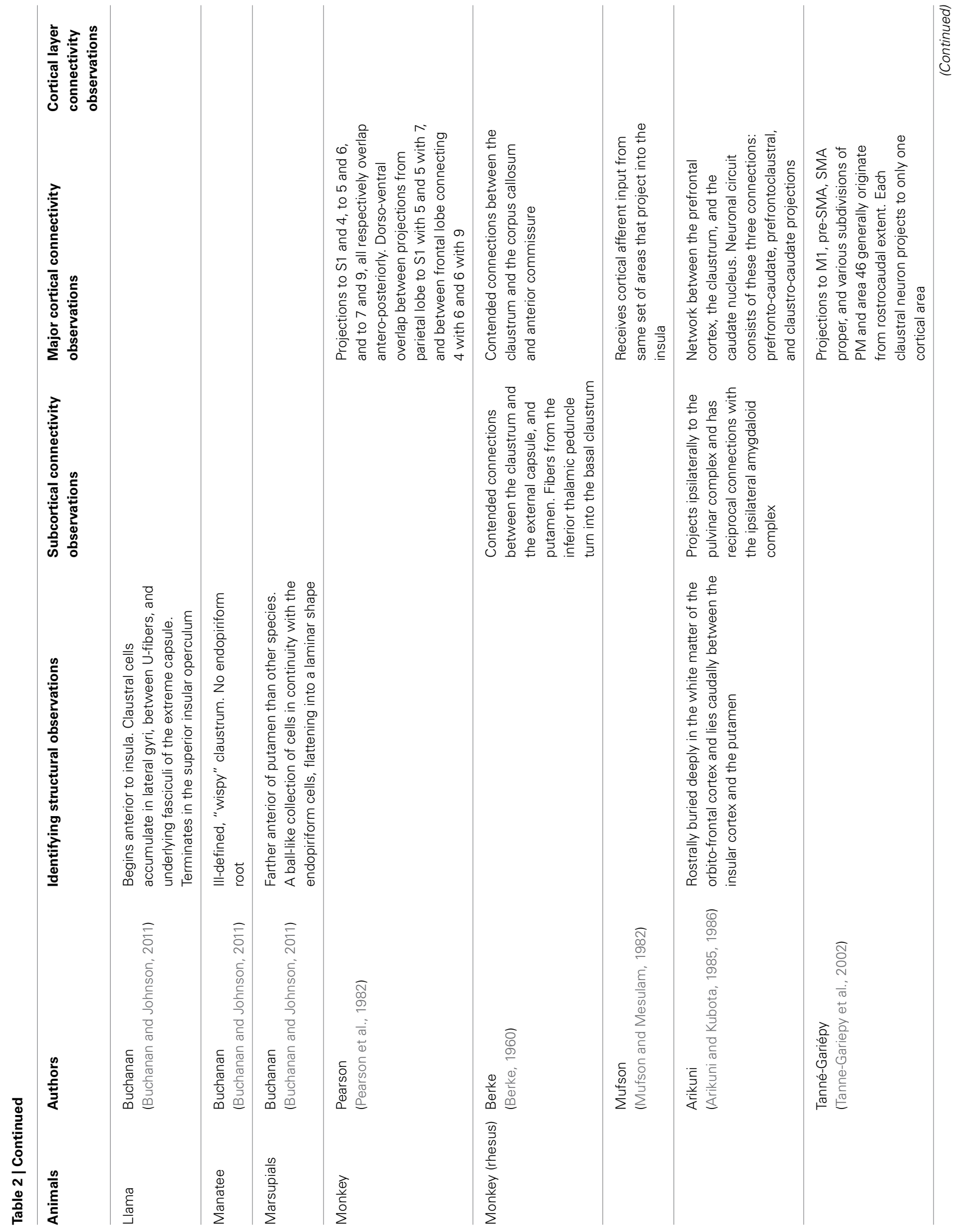




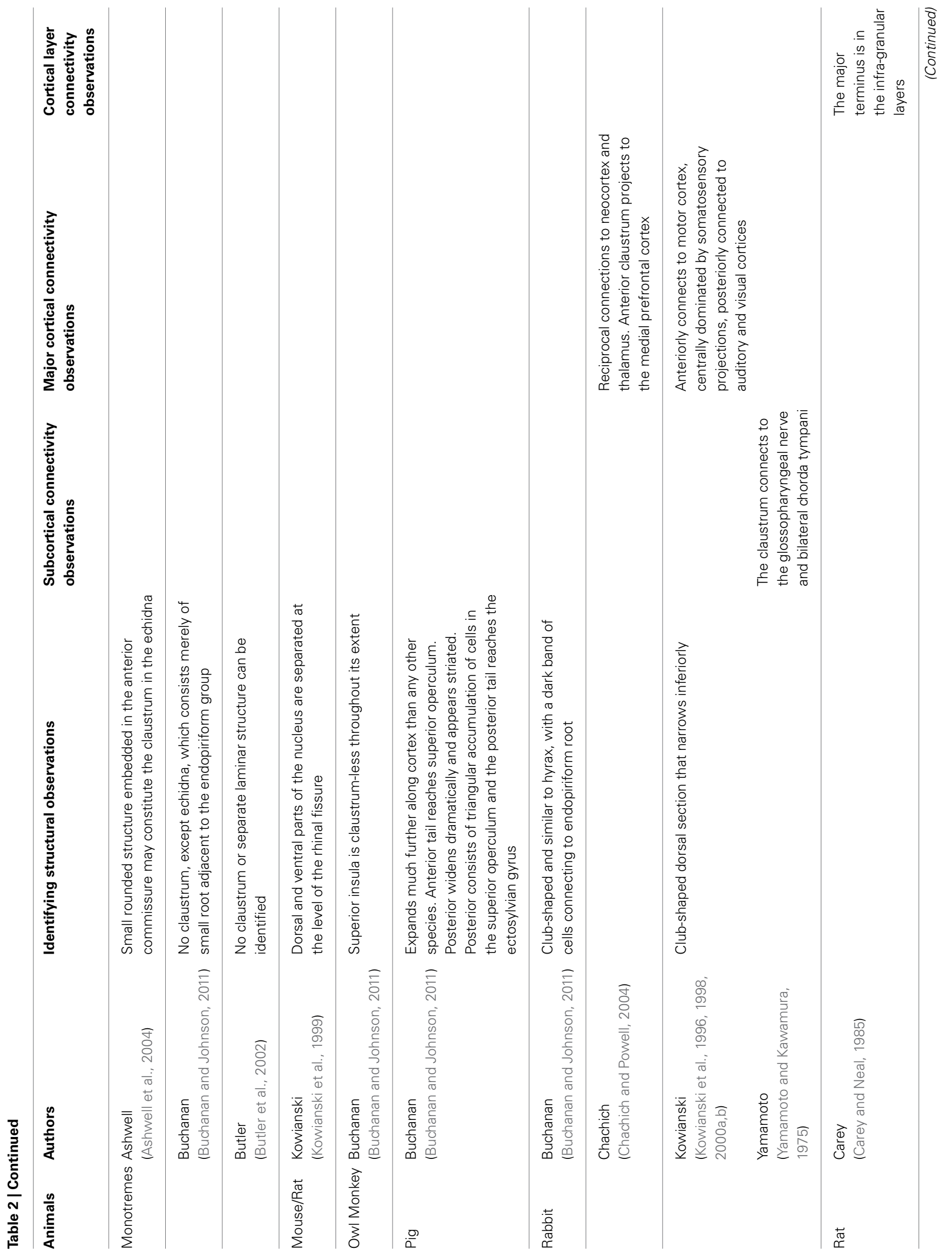




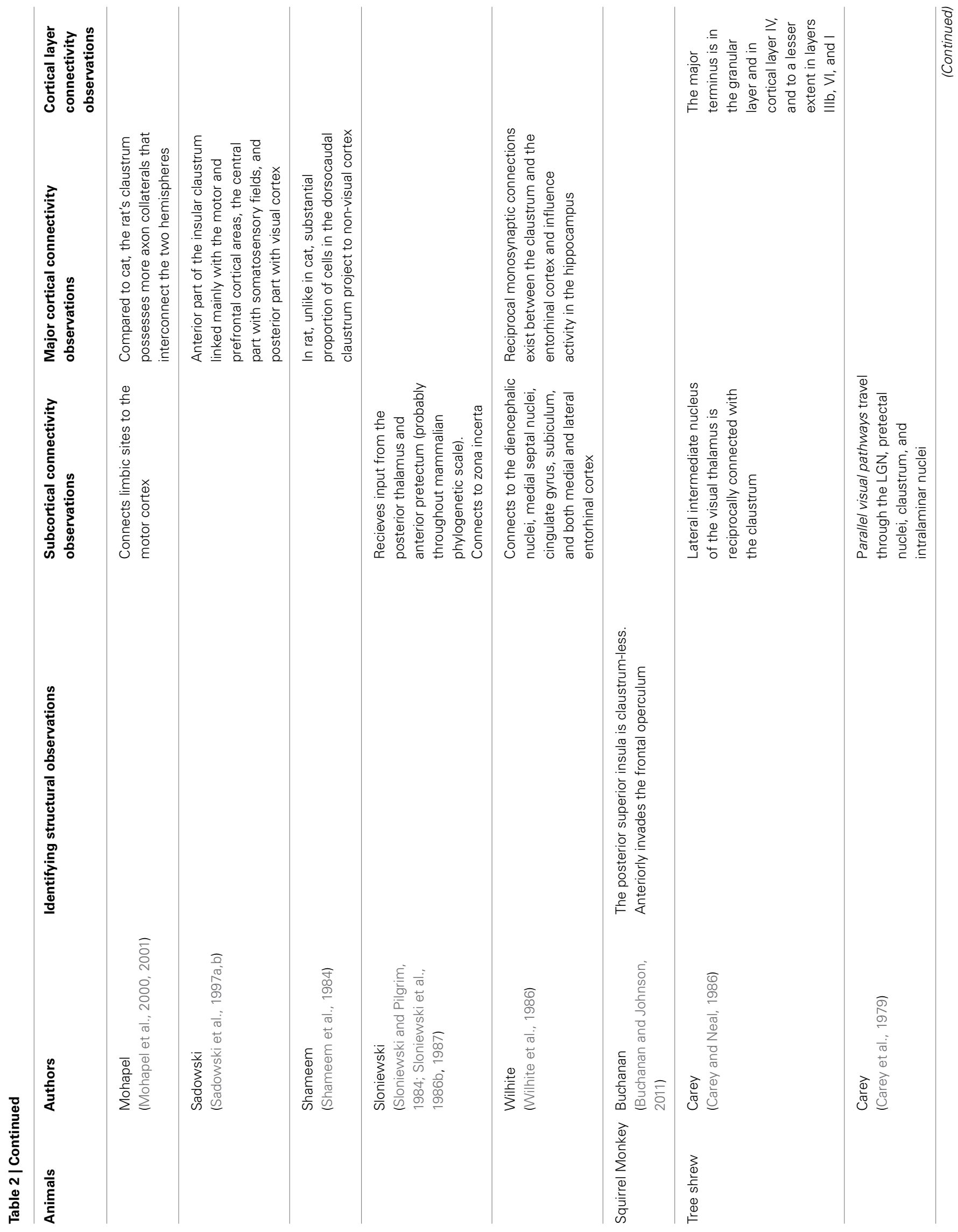




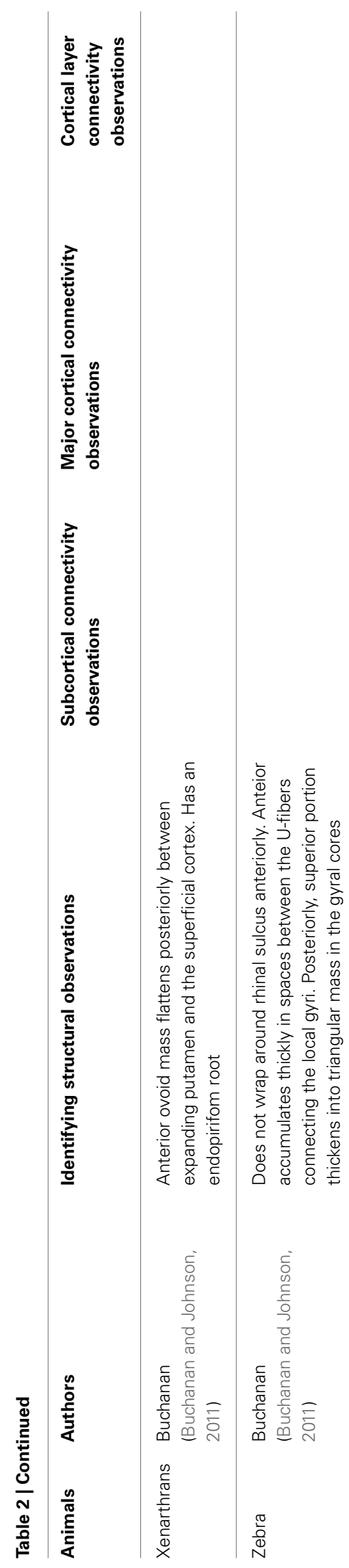

multiple sensory networks has led researchers to conclude that the claustrum functions primarily as a relay station between major networks (Minciacchi et al., 1995).

After centuries of analyzing individual connections, connectomics offers us the opportunity to analyze all connections to and from the claustrum and determine the weight of influence these have on the overall function of the structure. Such a gestalt view could shed light on the role of the claustrum, perhaps even providing a more precise definition of consciousness itself. Some researchers have eschewed a formal definition of consciousness, lest it result in biasing primary research or its results (Crick and Koch, 1990), while others list necessary components—such as an awareness of one's own physical and sentient existence (Craig, 2009), or varying awareness to unchanging stimuli (Blake et al., 2014) - but researchers seem to agree that conscious processes have access to a multitude of sensory information and some processing capacity. Characterization of a network or region in possession of these properties would almost certainly benefit from some graph theoretical calculation of the relative weights of network influence.

Still, reliance on newer investigative techniques will require an accumulation of many large studies in order to find certitude in our conclusions. In terms of DTI investigations, further knowledge of the anatomy of multi-orientational fiber populations may be necessary to increase accuracy; when axons are not oriented in a coherent fashion, so that then the voxel-averaged estimate of orientation cannot accurately summarize the orientation of the underlying fibers, continuity may be assumed between the fibers where there is none (Fernandez-Miranda et al., 2008a). Modern, in vivo, human imaging studies of the claustrum still suffer from low statistical power due to small sample sizes. Number of diffusion directions, voxel size, magnetic field strength, and eddy current correction will all need to be carefully controlled to obtain accurate results in a structure so small. In the long-term, parcellation algorithms (like FreeSurfer reconstruction; http://surfer. nmr.mgh.harvard.edu/) will need to become more precise if we hope to perform population-level analyses of small structures.

\section{STRUCTURAL MICRO-CONNECTOMICS}

"The central rationale for human connectomics builds on the premise that structural brain connectivity can serve as a basis for understanding brain dynamics and behavior" (Behrens and Sporns, 2012). Despite never having been subjected to network theory analysis, many observations of connections between the claustrum and individual regions or networks have helped establish some facts about claustral micro-connectomics. Hagmann et al. (2008) have applied community detection or modularity analysis to demonstrate-using diffusion imaging and resting state functional MRI-that a close relationship exists between structural connections and functional connections. Therefore, an analysis of the structural connections of the claustrum ought to allow us to hypothesize the functional role it may play in the networks to which it contributes. Functional imaging studies will be discussed in the following section.

Inputs to the claustrum arrive from a plethora of brain areas before being assimilated, integrated, and signals directed to the claustrum (Edelstein and Denaro, 2004). These functions 


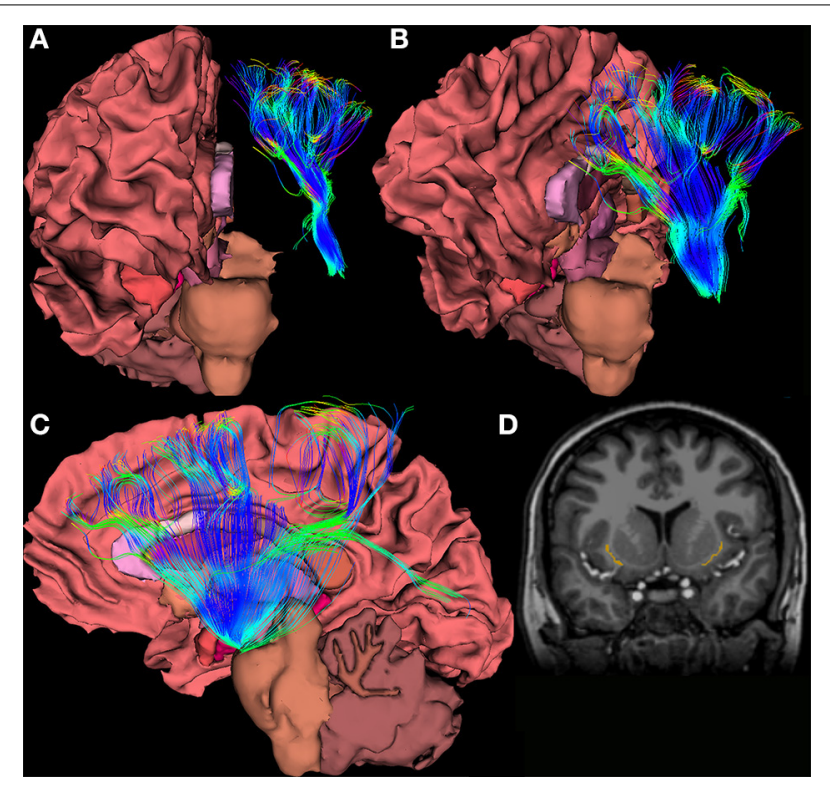

FIGURE 3 | (A-C) Views of a 64-direction diffusion tensor (DTI) fiber tract reconstruction from a 3.0 Telsa Siemens MRI Trio scanner in an example subject showing white matter pathways emanating from a the region of the human claustrum. (D) The image shows a binary label mask drawn of the left and right claustra on a T1-weighted image of the same subject.

allow rapid adaptation to nuanced changes in one's environment. Intra-claustral interactions, which may involve dendrodendritic synapses and networks of gap junction-linked neurons, are thought to be abundant (Smythies et al., 2012a,b), though the presence of connexin proteins would be a prerequisite for such networks which requires further exploration. Several authors have also found connections to the corpus callosum and anterior commissure (Berke, 1960; Milardi et al., 2013), although there are only one fifth as many contralateral projections as ipsilateral ones (Markowitsch et al., 1984). There is substantial evidence for claustrum-subcortical connections in the existing animal literature (for instance, in the cat, rat, and a range of insectivora, respectively; Kaufman and Rosenquist, 1985; Sloniewski et al., 1986a; Narkiewicz and Mamos, 1990; Dinopoulos et al., 1992), though there is a paucity of similar findings from ex vivo studies of humans. There is substantial evidence for claustrum-subcortical connections in the existing animal literature, though there is a paucity of similar findings from ex vivo studies of humans.

When multiple inputs converge onto the claustrum, this results in a new signal, which demonstrates integration (Edelstein and Denaro, 2004). It is particularly useful in cross-modal matching; for example, it is active when a subject sees and touches something, but not active when two items are seen or two items are touched (Arnow et al., 2002). Information can also be redirected throughout the brain by this structure (Edelstein and Denaro, 2004). In a study using macro-electrodes, the organization of somatic sensory, auditory, and visual projections to the claustrum were found to display heterotopic and multi-sensory convergence characteristics (Spector et al., 1970). Responses differ when the stimulus site or receiver site change, which indicates it is a non-homogeneous multisensory structure with three electrophysiologically distinct parts (Spector et al., 1970). Claustral neurons appear to discriminate and associate between intermodal and intra-modal sensory stimuli (Spector et al., 1974). The neurons of the striate cortex and pyramidal tract show decreased spontaneous firing during stimulation of the claustrum, which indicates it may play a role in regulating afferent sensory information (Edelstein and Denaro, 2004). Claustral cells themselves display very low spontaneous discharge (Spector et al., 1974). Connections to limbic regions seem to indicate that the claustrum possesses other functions beyond sensory integration. The amount of intra-modal branched inputs is higher in the claustrum than in relay nuclei, which indicates that the claustrum must do more than simply relay information (Minciacchi et al., 1995). Zingg et al. (2014) suggest that the claustrum may provide additional means of direct interaction between neural sub-networks. The structure and function of claustral connections may differ to some extent between genders. For example, the claustrum it is active during penile stimulation, but not clitoral stimulation (Georgiadis et al., 2009), and the level of activation corresponds to turgidity of the penis (Arnow et al., 2002). In fact, the claustrum and the brain stem are the only two brain regions active in males during sexual arousal, but inactive during competitive arousal (Redoute et al., 2000).

While the claustrum as a whole is known for multimodal processing, most neurons within it are not multisensory processors (Remedios et al., 2010). Because of this, it has been implied that perhaps claustrum synchronizes cortical regions that are responsible for bilaterally coordinated behaviors such as eye movements without actually changing the data being shuttled through itself (Smith and Alloway, 2010). Another hypothesis is that the claustrum seems to work to counterbalance the homunculus of the brain; overrepresented regions in S1 and V1 are relatively underrepresented in the claustrum and a preference exists for retinal periphery representation (LeVay and Sherk, 1981b; Minciacchi et al., 1995). Sherk and LeVay suggest that claustral efferents serve as inhibitors in order to shape the receptive field properties of cortical neurons (Sherk and LeVay, 1981; Shima et al., 1996). Furthermore, the claustrum may play a crucial role in plasticity and reorganization. Such a function would require the claustrum to recognize the unknown modular specific code carried by an afferent axon, or require claustral efferent to return to the same neuron (or neuronal group) that gave rise to the afferent axon (Smythies et al., 2012b).

Micro-connectomics of the claustrum was, until fairly recently, largely studied through retrograde tract-tracing, since the proximity of the claustrum to other areas makes placement of strictly claustral anatomical tracers incredibly imprecise. Unfortunately, retrograde tract-tracing requires clearly defined anatomical boundaries, which are still under investigation in the claustrum (Mathur et al., 2009).

\section{MACRO-CONNECTOMICS}

Sexuality studies may offer the easiest starting point for understanding the interactions between claustrocortical regions. Firstly, the claustrum is activated during penile, but not clitoral arousal (Georgiadis et al., 2009). Men and women tend to experience 
sexual arousal differently in response to visual stimuli, with one of the most notable differences being that visual stimulation is associated more with male arousal (Redoute et al., 2000; Hamann et al., 2004) — though this assumption has recently been questioned by Rupp and Wallen (2008). Arnow et al. (2002) suggest the claustrum may facilitate reflexive cross-modal transfer of visual input to imagined tactile (penile) stimulation. In both PET and PMRI investigations, claustrum activation corresponded to penile turgidity with statistical significance (Arnow et al., 2002; Stoleru et al., 2012). There is some question whether such activation is specific to sexual arousal, or may be indicative of more broad motivational processing; some imaging has shown claustral activation correlated with thirst, hunger, and emotional motivation (Stoleru et al., 2012).

A further breakdown of this relationship has been undertaken to separate sub-networks in which the claustrum participates during co-activation of the networks. It was demonstrated psychosexual arousal was characterized by lateral prefrontal cortex, superior parietal lobule, and medial and inferior frontal gyri (all bilaterally), while physiosexual arousal was characterized by anterior cingulate cortex (which the authors consider to be alternatively called ventromedial prefrontal cortex and medial orbitofrontal cortex) (Poeppl et al., 2014). The one region that was activated during both types of arousal was the claustrum. Furthermore, the right claustrum/insula was the only field shown to be involved in visual-tactile stimulation, but not activated in either pure tactile or pure visual stimulation (Hadjikhani and Roland, 1998). Due to observational analysis, researchers have long suspected that sexual stimuli and autonomic processes must be linked in the brain in a way that allows for two-way communication, and meta-analytic review suggests this link may be the claustrum and the putamen. Given its multimodal, cortical and cross-cortical connections, the claustrum seems like a viable candidate for cross-modal matching, such that modality-specific areas can communicate with the claustrum via unimodal connections, which can then be exchanged and altered to output a novel unimodal signal (Smythies et al., 2014). Additional functional imaging investigations of sexual arousal at ultra-high field and spatial resolution would provide confirmation of the psychophysiological role of the claustrum in concert with other brain regions believed to be different between males and females.

Claustral activation also occurs during identification of the use of a fluency heuristic, and may imply that the prioritization of certain inputs, along with the priming effect, may be driven or mediated despite its modest structural size (Volz et al., 2010). It has also been implicated in learning; the insula and claustrum are BOLD-activated during active, but not passive learning (Kersey and James, 2013). Given the prevalence of claustral abnormalities in memory disorders, the role of the claustrum in the creation of fluency heuristics may be due to its involvement in recall. If the conclusions about sensory integration are correct, an aclaustral subject could still respond to isolated stimuli, but would not be able to process complex ones or to coordinate the synchronization of inputs from multiple modalities (Crick and Koch, 2005).

In an fMRI study of musicians, Fauvel et al. (2014) found that the bilateral claustrum was functionally connected with the right inferior orbitofrontal gyrus at rest, and formed a resting-state executive control network. The authors suggested that the network integrates emotion aroused by the auditory stimuli in order to drive planning of future motor sequences that would continue to arouse the appropriate emotionalism in the music, although admitted that their functional conclusions were merely hypothetical.

Electrolytic lesions and ablations of the claustrum inhibit conditioned activity. Electrical stimulation can lead to salivation, tongue movements, blinking, and swallowing, and contralateral upper extremity motions, although results differ between stimulation of the claustral-putamen pathway and the claustralamygdala pathway (Edelstein and Denaro, 2004). Stimulation can also increase spinal reflexes. Pupillary dilation, licking, swallowing, shivering, and ear movement have been affected by claustral lesions in cats. Lesions of the left claustrum specifically lead to eyelid twitching, myoclonic jerking, and convulsive seizures, although one must remember lesion studies in small structures are more susceptible to downstream effects and other errors (Wada and Tsuchimochi, 1997).

\section{CLINICAL SYNDROMES ASSOCIATED WITH CLAUSTRAL DAMAGE AND DYSFUNCTION}

Table 3 lists the studies of clinical conditions where the claustrum has been implicated, along with the suspected means of claustral involvement. It is important to note that no clinical studies have been able to isolate the claustrum as the only region of involvement. This may support the idea that dysfunction in the claustrum results primarily in network disruption, rather than a specific functional deficit.

This diminutive structure has been investigated for its potential role in seizure generalization (Fernandez-Miranda et al., 2008 b). It is thought that epileptoform propagation from limbic sites may be linked to the motor cortex via the claustrum (Mohapel et al., 2000). A case study was conducted on a 12-yearold girl who presented with status epilepticus (multiple, repeated complex partial and myoclonic seizures occurred in the upper extremities and face with orofacial automatisms, eye deviation, and nystagmus) displaying psychotic behavior with agitation, severe cognitive impairment and temporary loss of vision, hearing and speech, as well as loss of orientation in time and place. Bilateral, strip-like lesions were discovered in T1 and T2 images of her claustrum and external capsule. The case indicates that lesions of the claustrum function more like gray matter disease than white matter disease. Additionally, the case study implies a functional correlation between seizures, behavioral state, and abnormalities in the claustrum (Sperner et al., 1996).

In contrast, unilateral removal of the claustrum did not lead to sensorimotor or cognitive impairment in patients receiving surgery for low-grade cerebral glioma (Duffau et al., 2007). So, perhaps the claustrum operates as part of a network or networks, rather than as the epicenter of a network.

The most common clinical association is the effect of the claustrum on memory. Claustral amyloid plaques accumulation has been implicated in the outcomes of Alzheimer's disease and aging (Morys et al., 1994; Fernandez-Miranda et al., 2008b). These amyloid deposits seem to cluster in the ventral claustrum, and may disrupt limbic connections (Morys et al., 1994). These 
Table 3 | Clinical syndromes with putative claustral involvement.

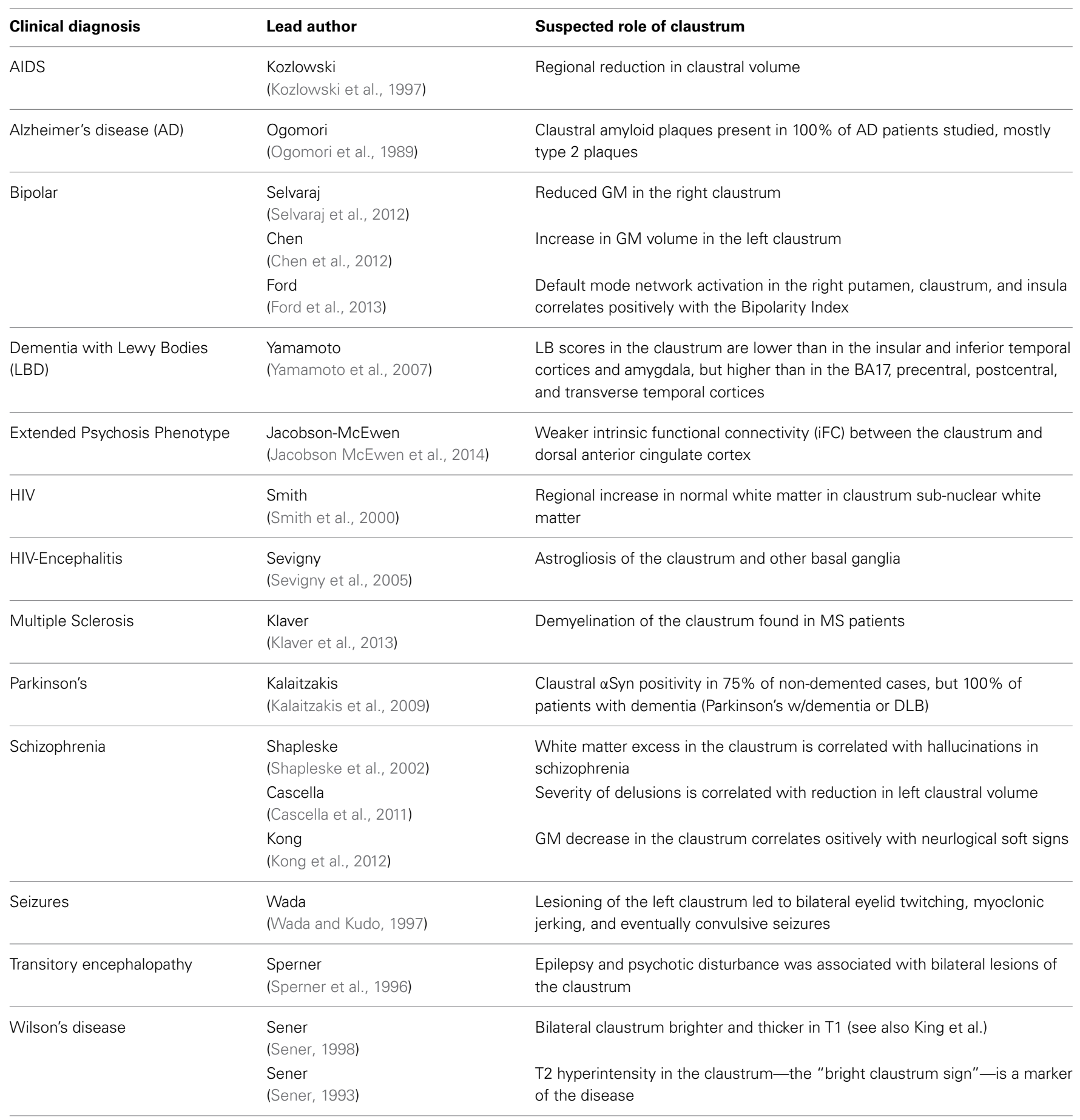

changes in the claustrum associated with aging, however, are subtle, appearing several years after those in the cerebral cortex become apparent. The claustrum has also been examined in cases of memory impairment associated with HIV, AIDS, Parkinson's, and Dementia with Lewy Bodies (DLB) (Kozlowski et al., 1997; Yamamoto et al., 2007; Smith et al., 2008; Kalaitzakis et al., 2009).

Other neurological conditions have been examined in the claustrum as well. Negative correlations between anhedonia and metabolism in the claustrum have been shown in both patients with unipolar depression and bipolar disorder (BD), which may be part of overall enlargement of the basal ganglia and increase in claustral GM volume in BD (Chen et al., 2011). Such factors may be the result of differences in pruning between diseased and healthy populations. Severity of delusions in schizophrenia is correlated with the reduction in left claustral volume (Cascella et al., 2011) and schizophrenia patients with hallucinations show signs 
of white matter excesses (Shapleske et al., 2002). The claustrum is alternately reported as spared from insular gliomas, or commonly found to be invaded by such tumors (Fernandez-Miranda et al., 2008a).

In Wilson's disease, abnormal brightness of T2-weighted images in the claustrum is considered one of the markers of the disease though its effects may be misattributed in the literature to its close neighbor, the putamen (Sener, 1993; King et al., 1996). In fact, Wilson himself cited the possible role of the claustrum in his initial description of the disease in 1912 (though he referred to it as "Progressive Lenticular Degeneration") (Wilson, 1912; Sener, 1993). The disease, which is invariably fatal without intervention, is caused by high copper levels - and sometimes iron levels - in the brain, particularly in the basal ganglia. Symptoms include bilateral tremors of the extremities, spasticity of the limbs and face, emaciation, dysphagia, dysarthria, emotionalism, and difficulty maintaining equilibrium (Wilson, 1912).

\section{CLAUSTRAL INVOLVEMENT IN NEURAL SYSTEMS}

Based on the summation of the micro-connectomics information gathered from centuries of histology, structural imaging, functional imaging, and clinical pathology studies, interactions have been proposed between the claustrum and every major sensory network. The extent to which these networks rely on the function of the claustrum, or to which the claustrum relies on information from these networks, remains to be investigated.

\section{VISUAL}

Claustral neurons are overwhelmingly binocular; $84 \%$ respond to stimuli from either eye, while only $40 \%$ of overall cortical neurons do (Sherk and LeVay, 1981). Responses in the claustrum to photic stimuli can be abolished by lesioning the lateral geniculate nucleus (LGN) (Edelstein and Denaro, 2004). V1 connections may not be reciprocal, as connections from $\mathrm{V} 1$ to the claustrum in macaques have not been reported (Crick and Koch, 2005).

Cortico-claustral connections from layer VI reach layer IV through the claustrum, creating an alternative route to the direct projection from layer VI to layer IV (LeVay and Sherk, 1981a). In the contralateral half of the visual field, the upper fields map to the caudal region of the claustrum, while the lower ones map rostrally. The far periphery can be mapped to the claustral surface, while the vertical meridian lies at the lower limit of the visual region. A single representation of the visual hemi-field exists as a unified map without discontinuities or duplications in the claustrum (LeVay and Sherk, 1981b).

\section{AUDITORY}

The medial ectosylvian gyrus (AII) relays information from the medial geniculate nucleus to the claustrum. In fact, ablation of the entire AII results in a lack of claustral response to MGN stimulation (Edelstein and Denaro, 2004).

\section{MOTOR}

Functional localization has not been demonstrated in the claustrum, so it is unlikely that the claustrum can influence specific muscular groups (Salerno et al., 1984).

The claustral loop to the striate cortex is involved in motion detection, but cannot discriminate the direction of motion
(Edelstein and Denaro, 2004). Most (approximately 70\%) of movement-related neurons increased discharge regardless of whether the motion was a push, a pull, or a turn, while only $16 \%$ were selective to one movement. This differs from the specificity of the motor cortex itself, in which about half of the neurons are responsive to a single motion (Shima et al., 1996). This may be indicative of a high degree of convergence of inputs. This lack of selectivity, along with the presence of inhibitory efferents, may suggest that the claustrum generally suppresses cortical activity immediately before the initiation of movement.

Projections to M1, pre-SMA, SMA-proper, and various subdivisions of PM and area 46 emanate from the entire rostro-caudal extent of the claustrum, with no distinct topographic or somatotopic organization. Along the dorso-ventral axis, however, these projections tend to originate in the dorsal or intermediate claustrum (Tanne-Gariepy et al., 2002). There is overlap of region of origin in most motor areas, but Pre-SMA and SMA-proper both uniquely show local segregation through inter-digitations. Area 46 also receives projections from the most ventral portion of the caudal claustrum, which sends minor projections to M1, the subareas of PM, pre-SMA, and SMA-proper (Tanne-Gariepy et al., 2002). M1 projections to the claustrum are then projected to $\mathrm{S} 1$, indicating a role in sensorimotor coordination (Smith et al., 2012).

In terms of oculomotor control, the mid-ventral claustrum receives projections from the frontal eye fields, and is organized topographically according to the size of saccades (Tanne-Gariepy et al., 2002).

\section{SOMATOSENSORY}

While there is general agreement on its heterotopic organization, there is no apparent somatotopic organization of the claustrum (Spector et al., 1974). Some somatic afferents to the claustrum are conveyed via the posterior spinal funiculi fibers. In addition, the anterior ectosylvian gyrus (SII) relays information from the ventral posterolateral nucleus (VPL) to the claustrum. Lesions in the VPL lead to reduced responsivity of the claustrum to skin stimulation. Marked claustral activity was noted in response to stimuli in somatic-vagal-tooth pulp. This may be indicative of trigeminal projections similar to those noted in other structures of the basal ganglia (Edelstein and Denaro, 2004).

\section{LOOKING TOWARD THE FUTURE}

Studying connectomics will improve the quality of human brain research. Firstly, connection profiles contain valuable information about a brain region. In fact, anatomical delineations between brain regions can be drawn out by examining which structural elements share similar long-range connections that differ from the connection profiles of other structures. Therefore, connectomics analysis could be a valuable tool in creating a reliable white matter atlas, a resource that does not currently exist. In fact, there is not even a universally agreed upon system of cortical parcellation (Sporns et al., 2005).

At present, the rapid proliferation of claustrum research seems to be mostly composed of macro-connectomics approaches, such as HARDI, DTI, and functional imaging. Shifting the focus, however, from micro to macro is not likely to solve all of the mysteries 
of the relationship between brain structure and function. Sporns and Tononi, in their noteworthy paper introducing the concept of the connectome, have argued that scientists would not be able to map structure to function in the human brain without a comprehensive connectional model (Sporns et al., 2005). A complete biophysical model of the human connectome, however, would "provide a unified, time-invariant, and readily available neuroinformatics resource that could be used in virtually all areas of experimental and theoretical neuroscience." Several maps of the human macro-scale structural connectome have been presented, (Irimia et al., 2012a,b; Barch et al., 2013; Ugurbil et al., 2013; Van Essen et al., 2013) with additional multimodal atlases forthcoming (Amunts et al., 2014). Yet, defining which clusters of connections comprise a sub-networks and determining the hierarchy of which networks depend on each other will take a lot of additional time and analysis of both the macro- and the micro-scale.

Micro-scale approaches to assessing connectivity have several shortcomings, although most of these can be mitigated by combining micro- and macro-scale approaches. Assessments of differences in network theory measures, such as betweenness centrality and assortativity, are difficult to translate into clinically relevant knowledge (Johansen-Berg, 2013). Histological attempts to study connectomics often neglect to take into account the difference in tissue size, behavior, and integrity caused by observation and intervention. Where histology tends to underestimate axonal properties, micro-structural observations from tractography data tend to be heavily influenced by the parameters of reconstruction chosen by the researcher (Assaf et al., 2013). Therefore, the combination of macro- and micro-scale connectomics allows for validation of statistics which tend to be particularly prone to error; crossing fibers, a perennial problem for tractography analysis, can be separated according to micro-structural characteristics such as axon diameter in order to increase the validity of diffusion data. Not only does this combination improve the accuracy of existing data analytic techniques, but a combinatory approach also opens the door to new, more complex and multi-scaled analyses. Localized data can be applied to estimation of conduction velocity, which helps in assigning network weight (JohansenBerg, 2013). Merging EEG and fMRI data with tractography and histology would allow us to take functional research conclusions out of the realm of correlation and start to understand the causation of the data patterns that have emerged in fMRI research. Uniting the two methods would undoubtedly generate further about the interplay of overlapping networks in the human brain (Assaf et al., 2013).

The claustrum appears ideally suited for synthesizing multimodal data because it appears to be the infrastructure through which sub-networks communicate. Since its discovery, its function was assumed to be connecting disparate cortical areas. However, using modern functional imaging methods, it is highly challenging to link behavioral function to such a diminutive region. Among the most relied upon approaches to inferring its function have been by assessing its connections, interactions, cell types, and shape to draw inferences which can be quantified.

For a combinatory connectomics analysis to succeed in the claustrum, changes will need to occur in the neuroimaging status quo. The claustrum literature is woefully lacking in vivo imaging, as well as human imaging. Regardless of species, sample sizes will need to increase dramatically, in order to help overcome the problem of individual differences that plagues neuroimaging data. Intra-individual differences should also be addressed, as there have been very few adjustments made to account for the growing evidence that connectivity is state-dependent. In small structures, tweaks in an imaging protocol can have a far more pronounced affect; therefore, it is vital that claustrum studies utilize the highest available resolution of MR images, and the highest number diffusion gradients. Including a claustrum label in any of the parcellation software atlases would drastically improve the reliability of the literature.

\section{CONCLUSIONS}

After centuries of research and much recent interest, a comprehensive theory of the claustrum's role in the brain and its sensory sub-networks remains lacking. Yet, the field of macro-scale connectomics-made possible through the refinement of neuroimaging and computational methods-provides a new basis for exploring intricate patterns of neural wiring and has already enabled broad-scale exploration of many major brain structures and pathways. The advancing sophistication of brain imaging and computational approaches is ideally suited for understanding the relative connectomics contributions of smaller brain regions which have only begun to be examined via brain mapping methods. Even the seemingly simple mathematical question of whether the claustrum is more crucial in inter- or intra- network connectivity remains unanswered and worthy of examination. Understanding whether the claustrum participates in each subnetwork to an equal quantitative extent would allow assessment of whether the structure performs a single function for each network or multiple functions, unique to each network. Comparison of claustral network metrics throughout human development could illuminate considerable information about the establishment and re-enforcement of inter- and intra-hemispheric communication. Finally, macro-connectomics analysis of a region of such growing popularity could set a leading precedent for investigating small structures and sub-structures of the brain.

In conclusion, the purpose of the claustrum-of interest to brain science for centuries - stands a chance of being unlocked through the use of macro-scale connectomics methods. While the study of neuronal connections is far from novel, the increasingly complex statistical algorithms for understanding the functions of network-wide interactions remain in their infancy. Furthermore, studies which combine microscopic detail with macro-scale investigation of multiple brain regions remain rare at present, if not unheard of. Such a wide scope of analysis would likely enhance the knowledge of almost any brain region; however, the putatively abstract and as yet unconfirmed role of the claustrum, beyond when considered in isolation, poses a valuable opportunity for connectomic analysis to break new ground and demonstrate its function by evaluating this stubbornly enigmatic structure across all scales. Likewise, multi-scale analysis of this under-appreciated brain region could demonstrate how much more robust our understanding becomes when networks and sub-networks are considered at all levels, rather than assuming that we can achieve 
this understanding through an additive process of examining individual micro-scale architecture. It is true that connectomic analysis is already enhancing our understanding of the brain. Yet, the advantages of these computational approaches seem underwhelming when merely used to recapitulate understanding of established networks. It remains unlikely-with contemporary technologies alone-that this mysterious strip of non-cortical, non-subcortical matter nestled deep within the cerebrum will ever be understood without applying connectomic network theory at the broadest possible scales.

\section{REFERENCES}

Amunts, K., Hawrylycz, M. J., Van Essen, D. C., Van Horn, J. D., Harel, N., Poline, J. B., et al. (2014). Interoperable atlases of the human brain. Neuroimage 99, 525-532. doi: 10.1016/j.neuroimage.2014.06.010

Arikuni, T., and Kubota, K. (1985). Claustral and amygdaloid afferents to the head of the caudate nucleus in macaque monkeys. Neurosci. Res. 2, 239-254. doi: 10.1016/0168-0102(85)90003-3

Arikuni, T., and Kubota, K. (1986). The organization of prefrontocaudate projections and their laminar origin in the macaque monkey: a retrograde study using HRP-gel. J. Comp. Neurol. 244, 492-510. doi: 10.1002/cne.9024 40407

Arnow, B. A., Desmond, J. E., Banner, L. L., Glover, G. H., Solomon, A., Polan, M. L., et al. (2002). Brain activation and sexual arousal in healthy, heterosexual males. Brain 125(Pt 5): 1014-1023. doi: 10.1093/brain/awf108

Ashwell, K. W., Hardman, C., and Paxinos, G. (2004). The claustrum is not missing from all monotreme brains. Brain Behav. Evol. 64, 223-241. doi: $10.1159 / 000080243$

Assaf, Y., Alexander, D. C., Jones, D. K., Bizzi, A., Behrens, T. E., Clark, C. A., et al. (2013). The CONNECT project: combining macro- and micro-structure. Neuroimage 80, 273-282. doi: 10.1016/j.neuroimage.2013.05.055

Barch, D. M., Burgess, G. C., Harms, M. P., Petersen, S. E., Schlaggar, B. L., Corbetta, M., et al. (2013). Function in the human connectome: taskfMRI and individual differences in behavior. Neuroimage 80, 169-189. doi: 10.1016/j.neuroimage.2013.05.033

Bayer, S. A., and Altman, J. (1991). Development of the endopiriform nucleus and the claustrum in the rat brain. Neuroscience 45, 391-412. doi: 10.1016/03064522(91) $90236-\mathrm{H}$

Behrens, T. E., and Sporns, O. (2012). Human connectomics. Curr. Opin. Neurobiol. 22, 144-153. doi: 10.1016/j.conb.2011.08.005

Berke, J. J. (1960). The claustrum, the external capsule and the extreme capsule of Macaca mulatto. J. Comp. Neurol. 115, 297-331. doi: 10.1002/cne.901150304

Blake, R., Brascamp, J., and Heeger, D. J. (2014). Can binocular rivalry reveal neural correlates of consciousness? Philos. Trans. R. Soc. Lond. B. Biol. Sci. 369:20130211. doi: 10.1098/rstb.2013.0211

Buchanan, K. J., and Johnson, J. I. (2011). Diversity of spatial relationships of the claustrum and insula in branches of the mammalian radiation. Ann. N.Y. Acad. Sci. 1225(Suppl 1), E30-E63. doi: 10.1111/j.1749-6632.2011.06022.x

Butler, A. B., Molnar, Z., and Manger, P. R. (2002). Apparent absence of claustrum in monotremes: implications for forebrain evolution in amniotes. Brain Behav. Evol. 60, 230-240. doi: 10.1159/000066698

Cao, Y., Whalen, S., Huang, J., Berger, K. L., and DeLano, M. C. (2003). Asymmetry of subinsular anisotropy by in vivo diffusion tensor imaging. Hum. Brain Mapp. 20, 82-90. doi: 10.1002/hbm.10130

Carey, R. G., Bear, M. F., and Diamond, I. T. (1980). The laminar organization of the reciprocal projections between the claustrum and striate cortex in the tree shrew, Tupaia glis. Brain Res. 184, 193-198. doi: 10.1016/0006-8993(80)90597-1

Carey, R. G., Fitzpatrick, D., and Diamond, I. T. (1979). Layer I of striate cortex of Tupaia glis and Galago senegalensis: projections from thalamus and claustrum revealed by retrograde transport of horseradish peroxidase. J. Comp. Neurol. 186, 393-437. doi: 10.1002/cne.901860306

Carey, R. G., and Neal, T. L. (1985). The rat claustrum: afferent and efferent connections with visual cortex. Brain Res. 329, 185-193. doi: 10.1016/00068993(85)90524-4

Carey, R. G., and Neal, T. L. (1986). Reciprocal connections between the claustrum and visual thalamus in the tree shrew (Tupaia glis). Brain Res. 386, 155-168. doi: 10.1016/0006-8993(86)90152-6
Cascella, N. G., Gerner, G. J., Fieldstone, S. C., Sawa, A., and Schretlen, D. J. (2011). The insula-claustrum region and delusions in schizophrenia. Schizophr. Res. 133, 77-81. doi: 10.1016/j.schres.2011.08.004

Chachich, M. E., and Powell, D. A. (2004). The role of claustrum in Pavlovian heart rate conditioning in the rabbit (Oryctolagus cuniculus): anatomical, electrophysiological, and lesion studies. Behav. Neurosci. 118, 514-525. doi: 10.1037/0735-7044.118.3.514

Chen, C. H., Suckling, J., Lennox, B. R., Ooi, C., and Bullmore, E. T. (2011). A quantitative meta-analysis of fMRI studies in bipolar disorder. Bipolar Disord. 13, 1-15. doi: 10.1111/j.1399-5618.2011.00893.x

Chen, Z., Cui, L., Li, M., Jiang, L., Deng, W., Ma, X., et al. (2012). Voxel based morphometric and diffusion tensor imaging analysis in male bipolar patients with first-episode mania. Prog. Neuropsychopharmacol. Biol. Psychiatry 36, 231-238. doi: 10.1016/j.pnpbp.2011.11.002

Craig, A. D. (2009). How do you feel-now? The anterior insula and human awareness. Nat. Rev. Neurosci. 10, 59-70. doi: 10.1038/nrn2555

Crick, F. C., and Koch, C. (2005). What is the function of the claustrum? Philos. Trans. R. Soc. Lond. B. Biol. Sci. 360, 1271-1279. doi: 10.1098/rstb. 2005.1661

Crick, F., and Koch, C. (1990). "Towards a neurobiological theory of consciousness," in Seminars in the Neurosciences (Philadelphia, PA: Saunders Scientific Publications).

Dinopoulos, A., Papadopoulos, G. C., Michaloudi, H., Parnavelas, J. G., Uylings, H. B., and Karamanlidis, A. N. (1992). Claustrum in the hedgehog (Erinaceus europaeus) brain: cytoarchitecture and connections with cortical and subcortical structures. J. Comp. Neurol. 316, 187-205. doi: 10.1002/cne.903 160205

Druga, R. (2014). "Structure and connections of the claustrum," in The Claustrum: Structural, Functional, and Clinical Neuroscience, Vol. 1, eds J. Smythies, L. Edelstein, and V. S. Ramachandran (San Diego, CA: Academic Press), $29-84$.

Duffau, H., Mandonnet, E., Gatignol, P., and Capelle, L. (2007). Functional compensation of the claustrum: lessons from low-grade glioma surgery. J. Neurooncol. 81, 327-329. doi: 10.1007/s11060-006-9236-8

Edelstein, L. R., and Denaro, F. J. (2004). The claustrum: a historical review of its anatomy, physiology, cytochemistry and functional significance. Cell. Mol. Biol. (Noisy-le-grand). 50, 675-702. doi: 10.1170/T558

Eling, P. (1994). Reader in the History of Aphasia: From Franz Gall to Norman Geschwind. Philadelphia, PA: John Benjamins Publishing. doi: 10.1075/cipl.4

Fauvel, B., Groussard, M., Chetelat, G., Fouquet, M., Landeau, B., Eustache, F., et al. (2014). Morphological brain plasticity induced by musical expertise is accompanied by modulation of functional connectivity at rest. Neuroimage 90, 179-188. doi: 10.1016/j.neuroimage.2013.12.065

Fernandez-Miranda, J. C., Pathak, S., Engh, J., Jarbo, K., Verstynen, T., Yeh, F. C., et al. (2012). High-definition fiber tractography of the human brain: neuroanatomical validation and neurosurgical applications. Neurosurgery 71 , 430-453. doi: 10.1227/NEU.0b013e3182592faa

Fernandez-Miranda, J. C., Rhoton, A. L. Jr., Alvarez-Linera, J., Kakizawa, Y., Choi, C., and de Oliveira, E. P. (2008a). Three-dimensional microsurgical and tractographic anatomy of the white matter of the human brain. Neurosurgery 62(6 Suppl 3), 989-1026. discussion: 1026-1028. doi: 10.1227/01.neu.0000333767.05328.49

Fernandez-Miranda, J. C., Rhoton, A. L. Jr., Kakizawa, Y., Choi, C., and AlvarezLinera, J. (2008b). The claustrum and its projection system in the human brain: a microsurgical and tractographic anatomical study. J. Neurosurg. 108, 764-774. doi: 10.3171/JNS/2008/108/4/0764

Ford, K. A., Theberge, J., Neufeld, R. J., Williamson, P. C., and Osuch, E. A. (2013). Correlation of brain default mode network activation with bipolarity index in youth with mood disorders. J. Affect. Disord. 150, 1174-1178. doi: 10.1016/j.jad.2013.05.088

Georgiadis, J. R., Reinders, A. A., Paans, A. M., Renken, R., and Kortekaas, R. (2009). Men versus women on sexual brain function: prominent differences during tactile genital stimulation, but not during orgasm. Hum. Brain Mapp. 30, 3089-3101. doi: 10.1002/hbm.20733

Grasby, K., and Talk, A. (2013). The anterior claustrum and spatial reversal learning in rats. Brain Res. 1499, 43-52. doi: 10.1016/j.brainres.2013.01.014

Hadjikhani, N., and Roland, P. E. (1998). Cross-modal transfer of information between the tactile and the visual representations in the human brain: a positron emission tomographic study. J. Neurosci. 18, 1072-1084. 
Hagmann, P., Cammoun, L., Gigandet, X., Meuli, R., Honey, C. J., Wedeen, V., et al. (2008). Mapping the structural core of human cerebral cortex. PLoS Biol. 6, 1479-1493. doi: 10.1371/journal.pbio.0060159

Hamann, S., Herman, R. A., Nolan, C. L., and Wallen, K. (2004). Men and women differ in amygdala response to visual sexual stimuli. Nat. Neurosci. 7, 411-416. doi: $10.1038 / \mathrm{nn} 1208$

Irimia, A., Chambers, M. C., Torgerson, C. M., Filippou, M., Hovda, D. A., Alger, J. R., et al. (2012a). Patient-tailored connectomics visualization for the assessment of white matter atrophy in traumatic brain injury. Front. Neurol. 3:10. doi: 10.3389/fneur.2012.00010

Irimia, A., Chambers, M. C., Torgerson, C. M., and Horn, J. D. (2012b). Circular representation of human cortical networks for subject and population-level connectomic visualization. Neuroimage 60, 1340-1351. doi: 10.1016/j.neuroimage.2012.01.107

Jacobson McEwen, S. C., Connolly, C. G., Kelly, A. M., Kelleher, I., O’Hanlon, E., Clarke, M., et al. (2014). Resting-state connectivity deficits associated with impaired inhibitory control in non-treatment-seeking adolescents with psychotic symptoms. Acta Psychiatr. Scand. 129, 134-142. doi: 10.1111/acps. 12141

Johansen-Berg, H. (2013). Human connectomics-What will the future demand? Neuroimage 80, 541-544. doi: 10.1016/j.neuroimage.2013.05.082

Kalaitzakis, M. E., Pearce, R. K., and Gentleman, S. M. (2009). Clinical correlates of pathology in the claustrum in Parkinson's disease and dementia with Lewy bodies. Neurosci. Lett. 461, 12-15. doi: 10.1016/j.neulet.2009.05.083

Kapakin, S. (2011). The claustrum: three-dimensional reconstruction, photorealistic imaging, and stereotactic approach. Folia Morphol. (Warsz). 70, 228-234. Available online at: http://czasopisma.viamedica.pl/fm/article/ download/19276/15169

Kaufman, E. F., and Rosenquist, A. C. (1985). Efferent projections of the thalamic intralaminar nuclei in the cat. Brain Res. 335, 257-279. doi: 10.1016/00068993(85)90478-0

Kersey, A. J., and James, K. H. (2013). Brain activation patterns resulting from learning letter forms through active self-production and passive observation in young children. Front. Psychol. 4:567. doi: 10.3389/fpsyg.2013.00567

King, A. D., Walshe, J. M., Kendall, B. E., Chinn, R. J., Paley, M. N., Wilkinson, I. D., et al. (1996). Cranial MR imaging in Wilson's disease. AJR Am. J. Roentgenol. 167, 1579-1584. doi: 10.2214/ajr.167.6.8956601

Klaver, R., De Vries, H. E., Schenk, G. J., and Geurts, J. J. (2013). Grey matter damage in multiple sclerosis: a pathology perspective. Prion 7, 66-75. doi: 10.4161/pri.23499

Kong, L., Bachmann, S., Thomann, P. A., Essig, M., and Schroder, J. (2012). Neurological soft signs and gray matter changes: a longitudinal analysis in first-episode schizophrenia. Schizophr. Res. 134, 27-32. doi: 10.1016/j.schres.2011.09.015

Konukoglu, E., van der Kouwe, A., Sabuncu, M. R., and Fischl, B. (2013). Examplebased restoration of high-resolution magnetic resonance image acquisitions. Med. Image Comput. Comput. Assist. Interv. 8149, 131-138. doi: 10.1007/9783-642-40811-3_17

Kowianski, P., Dziewiatkowski, J., Karwacki, Z., and Morys, J. (2000a). The different patterns of maturation of the claustrocortical connections in a rabbit. Folia Morphol. (Warsz). 59, 193-200.

Kowianski, P., Dziewiatkowski, J., Kowianska, J., and Morys, J. (1999). Comparative anatomy of the claustrum in selected species: a morphometric analysis. Brain Behav. Evol. 53, 44-54. doi: 10.1159/000006581

Kowianski, P., Morys, J., Dziewiatkowski, J., Karwacki, Z., Bobek-Billewicz, B., and Narkiewicz, O. (1996). Neurons of the claustrum projecting to the motor and somatosensory cortex in the rabbit-assessment with the method of retrograde transport of fluorescent tracers. Folia Morphol. (Warsz). 55, 350-352.

Kowianski, P., Morys, J., Dziewiatkowski, J., Karwacki, Z., and Wisniewski, H. M. (2000b). The combined retrograde transport and unbiased stereological study of the claustrocortical connections in the rabbit. Ann. Anat. 182, 111-122. doi: 10.1016/S0940-9602(00)80068-0

Kowianski, P., Morys, J., Karwacki, Z., Dziewiatkowski, J., and Narkiewicz, O. (1998). The cortico-related zones of the rabbit claustrum-study of the claustrocortical connections based on the retrograde axonal transport of fluorescent tracers. Brain Res. 784, 199-209. doi: 10.1016/S0006-8993(97)01326-7

Kozlowski, P. B., Brudkowska, J., Kraszpulski, M., Sersen, E. A., Wrzolek, M. A., Anzil, A. P., et al. (1997). Microencephaly in children congenitally infected with human immunodeficiency virus-a gross-anatomical morphometric study. Acta Neuropathol. 93, 136-145. doi: 10.1007/s004010050594

Landau, E. (1919). The comparative anatomy of the nucleus amygdalae, the claustrum and the insular cortex. J. Anat. 53(Pt 4), 351-360.

Lander, E. S., Linton, L. M., Birren, B., Nusbaum, C., Zody, M. C., Baldwin, J., et al. (2001). Initial sequencing and analysis of the human genome. Nature 409, 860-921. doi: 10.1038/35057062

LeVay, S. (1986). Synaptic organization of claustral and geniculate afferents to the visual cortex of the cat. J. Neurosci. 6, 3564-3575.

LeVay, S., and Sherk, H. (1981a). The visual claustrum of the cat. I. Structure and connections. J. Neurosci 1, 956-980.

LeVay, S., and Sherk, H. (1981b). The visual claustrum of the cat. II. The visual field map. J Neurosci 1, 981-992.

Markowitsch, H. J., Irle, E., Bang-Olsen, R., and Flindt-Egebak, P. (1984). Claustral efferents to the cat's limbic cortex studied with retrograde and anterograde tracing techniques. Neuroscience 12, 409-425. doi: 10.1016/0306-4522(84)90062-9

Mathur, B. N., Caprioli, R. M., and Deutch, A. Y. (2009). Proteomic analysis illuminates a novel structural definition of the claustrum and insula. Cereb. Cortex 19, 2372-2379. doi: 10.1093/cercor/bhn253

Meng, H., Zhang, Z., Geng, H., Lin, X., Feng, L., Teng, G., et al. (2012) Development of the subcortical brain structures in the second trimester: assessment with 7.0-T MRI. Neuroradiology 54, 1153-1159. doi: 10.1007/s00234-0121069-x

Milardi, D., Bramanti, P., Milazzo, C., Finocchio, G., Arrigo, A., Santoro, G., et al. (2013). Cortical and subcortical connections of the human claustrum revealed in vivo by constrained spherical deconvolution tractography. Cereb Cortex. doi: 10.1093/cercor/bht231. [Epub ahead of print].

Minciacchi, D., Granato, A., Antonini, A., Tassinari, G., Santarelli, M., Zanolli, L., et al. (1995). Mapping subcortical extrarelay afferents onto primary somatosensory and visual areas in cats. J. Comp. Neurol. 362, 46-70. doi: $10.1002 /$ cne. 903620104

Minciacchi, D., Molinari, M., Bentivoglio, M., and Macchi, G. (1985). The organization of the ipsi- and contralateral claustrocortical system in rat with notes on the bilateral claustrocortical projections in cat. Neuroscience 16, 557-576. doi: 10.1016/0306-4522(85)90192-7

Mohapel, P., Hannesson, D. K., Armitage, L. L., Gillespie, G. W., and Corcoran, M. E. (2000). Claustral lesions delay amygdaloid kindling in the rat. Epilepsia 41, 1095-1101. doi: 10.1111/j.1528-1157.2000.tb00313.x

Mohapel, P., Zhang, X., Gillespie, G. W., Chlan-Fourney, J., Hannesson, D. K., Corley, S. M., et al. (2001). Kindling of claustrum and insular cortex: comparison to perirhinal cortex in the rat. Eur. J. Neurosci. 13, 1501-1519. doi: 10.1046/j.0953-816x.2001.01532.x

Molnár, Z. (2004). Thomas Willis (1621-1675), the founder of clinical neuroscience. Nature Reviews Neuroscience 5, 329-335. doi: 10.1038/nrn1369

Morys, J., Narkiewicz, O., Maciejewska, B., Wegiel, J., and Wisniewski, H. M. (1994). Amyloid deposits and loss of neurones in the claustrum of the aged dog. Neuroreport 5, 1825-1828. doi: 10.1097/00001756-199409080-00035

Mufson, E. J., and Mesulam, M. M. (1982). Insula of the old world monkey. II: Afferent cortical input and comments on the claustrum. J. Comp. Neurol. 212, 23-37. doi: 10.1002/cne.902120103

Naghavi, H. R., Eriksson, J., Larsson, A., and Nyberg, L. (2007). The claustrum/insula region integrates conceptually related sounds and pictures. Neurosci. Lett. 422, 77-80. doi: 10.1016/j.neulet.2007.06.009

Narkiewicz, O., and Mamos, L. (1990). Relation of the insular claustrum to the neocortex in Insectivora. J. Hirnforsch. 31, 623-633.

Ogomori, K., Kitamoto, T., Tateishi, J., Sato, Y., Suetsugu, M., and Abe, M. (1989). Beta-protein amyloid is widely distributed in the central nervous system of patients with Alzheimer's disease. Am. J. Pathol. 134, 243-251.

Parent, A. (2012). The history of the basal ganglia: the contribution of karl friedrich burdach. Neurosci. Med. 3, 374-379. doi: 10.4236/nm.2012.34046

Park, S., Tyszka, J. M., and Allman, J. M. (2012). The claustrum and insula in microcebus murinus: a high resolution diffusion imaging study. Front. Neuroanat. 6:21. doi: 10.3389/fnana.2012.00021

Patzke, N., Innocenti, G. M., and Manger, P. R. (2014). The claustrum of the ferret: afferent and efferent connections to lower and higher order visual cortical areas. Front. Syst. Neurosci. 8:57. doi: 10.3389/fnsys.2014.00031

Pearson, R. C., Brodal, P., Gatter, K. C., and Powell, T. P. (1982). The organization of the connections between the cortex and the claustrum in the monkey. Brain Res. 234, 435-441. doi: 10.1016/0006-8993(82)90883-6 
Pirone, A., Cozzi, B., Edelstein, L., Peruffo, A., Lenzi, C., Quilici, F., et al. (2012). Topography of Gng2- and NetrinG2-expression suggests an insular origin of the human claustrum. PLoS ONE 7:e44745. doi: 10.1371/journal.pone. 0044745

Poeppl, T. B., Langguth, B., Laird, A. R., and Eickhoff, S. B. (2014). The functional neuroanatomy of male psychosexual and physiosexual arousal: a quantitative meta-analysis. Hum. Brain Mapp. 35, 1404-1421. doi: 10.1002/hbm. 22262

Puelles, L. (2014). "Development and evolution of the claustrum," in The Claustrum: Structural, Functional, and Clinical Neuroscience, eds J. Smythies, L. Edelstein, and V. S. Ramachandran (San Diego, CA: Academic Press), 1.

Redoute, J., Stoleru, S., Gregoire, M. C., Costes, N., Cinotti, L., Lavenne, F., et al. (2000). Brain processing of visual sexual stimuli in human males. Hum. Brain Mapp. 11, 162-177. doi: 10.1002/1097-0193

Remedios, R., Logothetis, N. K., and Kayser, C. (2010). Unimodal responses prevail within the multisensory claustrum. J. Neurosci. 30, 12902-12907. doi: 10.1523/JNEUROSCI.2937-10.2010

Rupp, H. A., and Wallen, K. (2008). Sex differences in response to visual sexual stimuli: a review. Arch. Sex. Behav. 37, 206-218. doi: 10.1007/s10508-0079217-9

Sadowski, M., Morys, J., Jakubowska-Sadowska, K., and Narkiewicz, O. (1997a). Rat's claustrum shows two main cortico-related zones. Brain Res. 756, 147-152. doi: 10.1016/S0006-8993(97)00135-2

Sadowski, M., Morys, J., Jakubowska-Sadowska, K., and Narkiewicz, O. (1997b). Some claustral neurons projecting to various neocortical areas show morphological differences. Folia Morphol. (Warsz). 56, 65-76.

Salerno, M. T., Cortimiglia, R., Crescimanno, G., and Amato, G. (1989). Effect of claustrum activation on the spontaneous unitary activity of frontal eye field neurons in the cat. Neurosci. Lett. 98, 299-304. doi: 10.1016/03043940(89)90418-7

Salerno, M. T., Cortimiglia, R., Crescimanno, G., Amato, G., and Infantellina, F. (1984). Effects of claustrum stimulation on spontaneous bioelectrical activity of motor cortex neurons in the cat. Exp. Neurol. 86, 227-239. doi: 10.1016/00144886(84)90183-3

Salerno, M. T., Zagami, M. T., Cortimiglia, R., and Infantellina, F. (1981). The action of iontophoretically applied acetylcholine and dopamine on single claustrum neurones in the cat. Neuropharmacology 20, 895-899. doi: 10.1016/00283908(81)90085-X

Selvaraj, S., Arnone, D., Job, D., Stanfield, A., Farrow, T. F., Nugent, A. C., et al. (2012). Grey matter differences in bipolar disorder: a meta-analysis of voxelbased morphometry studies. Bipolar Disord. 14, 135-145. doi: 10.1111/j.13995618.2012.01000.x

Sener, R. N. (1993). The claustrum on MRI: normal anatomy, and the bright claustrum as a new sign in Wilson's disease. Pediatr. Radiol. 23, 594-596. doi: 10.1007/BF02014975

Sener, R. N. (1998). Lesions affecting the claustrum. Comput. Med. Imaging Graph. 22, 57-61. doi: 10.1016/S0895-6111(97)00043-8

Sevigny, J. J., Chin, S. S., Milewski, Y., Albers, M. W., Gordon, M. L., and Marder, K. (2005). HIV encephalitis simulating Huntington's disease. Mov. Disord. 20, 610-613. doi: 10.1002/mds.20379

Shameem, N., Sanderson, K., and Dreher, B. (1984). Claustral afferents to the rat's visual cortex. Neurosci. Lett. 49, 247-252. doi: 10.1016/0304-3940(84) 90297-0

Shapleske, J., Rossell, S. L., Chitnis, X. A., Suckling, J., Simmons, A., Bullmore, E. T., et al. (2002). A computational morphometric MRI study of schizophrenia: effects of hallucinations. Cereb. Cortex 12, 1331-1341. doi: 10.1093/cercor/12.12.1331

Sherk, H., and LeVay, S. (1981). The visual claustrum of the cat. III. Receptive field properties. J. Neurosci. 1, 993-1002.

Shima, K., Hoshi, E., and Tanji, J. (1996). Neuronal activity in the claustrum of the monkey during performance of multiple movements. J. Neurophysiol. 76, 2115-2119.

Sloniewski, P., and Pilgrim, C. (1984). Claustro-neocortical connections in the rat as demonstrated by retrograde tracing with Lucifer yellow. Neurosci. Lett. 49, 29-32. doi: 10.1016/0304-3940(84)90131-9

Sloniewski, P., Pilgrim, C., Morys, J., and Narkiewicz, O. (1987). Projections from the pretectothalamic border area to the somatosensory part of the claustrum: an autoradiographic study. Acta Neurobiol. Exp. (Wars). 47, 179-182.

Sloniewski, P., Usunoff, K. G., and Pilgrim, C. (1986a). Diencephalic and mesencephalic afferents of the rat claustrum. Anat. Embryol. (Berl.) 173, 401-411. doi: 10.1007/BF00318925

Sloniewski, P., Usunoff, K. G., and Pilgrim, C. (1986b). Retrograde transport of fluorescent tracers reveals extensive ipsi- and contralateral claustrocortical connections in the rat. J. Comp. Neurol. 246, 467-477. doi: 10.1002/cne.9024 60405

Smith, C. A., Stebbins, G. T., Bartt, R. E., Kessler, H. A., Adeyemi, O. M., Martin, E., et al. (2008). Gender effects on HIV-associated white matter alterations: a voxel-wise DTI study. Brain Imaging Behav. 2, 177-191. doi: 10.1007/s11682008-9024-5

Smith, J. B., and Alloway, K. D. (2010). Functional specificity of claustrum connections in the rat: interhemispheric communication between specific parts of motor cortex. J. Neurosci. 30, 16832-16844. doi: 10.1523/JNEUROSCI.443810.2010

Smith, J. B., Radhakrishnan, H., and Alloway, K. D. (2012). Rat claustrum coordinates but does not integrate somatosensory and motor cortical information. J. Neurosci. 32, 8583-8588. doi: 10.1523/JNEUROSCI.152412.2012

Smith, K. M., Crandall, K. A., Kneissl, M. L., and Navia, B. A. (2000). PCR detection of host and HIV-1 sequences from archival brain tissue. J. Neurovirol. 6 , 164-171. doi: 10.3109/13550280009013160

Smythies, J., Edelstein, L., and Ramachandran, V. (2012a). The functional anatomy of the claustrum: the net that binds. WebmedCentral NEUROSCIENCES 3:WMC003182. doi: 10.9754/journal.wmc.2012.003182

Smythies, J., Edelstein, L., and Ramachandran, V. (2012b). Hypotheses relating to the function of the claustrum. Front. Integr. Neurosci. 6:53. doi: 10.3389/fnint.2012.00053

Smythies, J., Edelstein, L., and Ramachandran, V. (2014). Hypotheses relating to the function of the claustrum II: does the claustrum use frequency codes? Front. Integr. Neurosci. 8:7. doi: 10.3389/fnint.2014.00007

Spector, I., Hassmannova, J., and Albe-Fessard, D. (1974). Sensory properties of single neurons of cat's claustrum. Brain Res. 66, 39-65. doi: 10.1016/00068993(74)90077-8

Spector, I., Hassmannova, Y., and Albe-Fessard, D. (1970). A macrophysiological study of functional organization of the claustrum. Exp. Neurol. 29, 31-51. doi: 10.1016/0014-4886(70)90035-X

Sperner, J., Sander, B., Lau, S., Krude, H., and Scheffner, D. (1996). Severe transitory encephalopathy with reversible lesions of the claustrum. Pediatr. Radiol. 26, 769-771. doi: 10.1007/BF01396197

Sporns, O., Tononi, G., and Kotter, R. (2005). The human connectome: a structural description of the human brain. PLoS Comput. Biol. 1:e42. doi: 10.1371/journal.pcbi.0010042

Stoleru, S., Fonteille, V., Cornelis, C., Joyal, C., and Moulier, V. (2012). Functional neuroimaging studies of sexual arousal and orgasm in healthy men and women: a review and meta-analysis. Neurosci. Biobehav. Rev. 36, 1481-1509. doi: 10.1016/j.neubiorev.2012.03.006

Talairach, J., and Tournoux, P. (1988). Co-Planar Stereotaxic Atlas of the Human Brain. New York, NY: Thieme.

Tanne-Gariepy, J., Boussaoud, D., and Rouiller, E. M. (2002). Projections of the claustrum to the primary motor, premotor, and prefrontal cortices in the macaque monkey. J. Comp. Neurol. 454, 140-157. doi: 10.1002/cne. 10425

Tsumoto, T., and Suda, K. (1982). Effects of stimulation of the dorsocaudal claustrum on activities of striate cortex neurons in the cat. Brain Res. 240, 345-349. doi: 10.1016/0006-8993(82)90233-5

Ugurbil, K., Xu, J., Auerbach, E. J., Moeller, S., Vu, A. T., Duarte-Carvajalino, J. M., et al. (2013). Pushing spatial and temporal resolution for functional and diffusion MRI in the Human Connectome Project. Neuroimage 80, 80-104. doi: 10.1016/j.neuroimage.2013.05.012

Van Essen, D. C., Smith, S. M., Barch, D. M., Behrens, T. E., Yacoub, E., Ugurbil, K., et al. (2013). The WU-Minn human connectome project: an overview. Neuroimage 80, 62-79. doi: 10.1016/j.neuroimage.2013.05.041

Volz, K. G., Schooler, L. J., and von Cramon, D. Y. (2010). It just felt right: the neural correlates of the fluency heuristic. Conscious. Cogn. 19, 829-837. doi: 10.1016/j.concog.2010.05.014 
Wada, J. A., and Kudo, T. (1997). Involvement of the claustrum in the convulsive evolution of temporal limbic seizure in feline amygdaloid kindling. Electroencephalogr. Clin. Neurophysiol. 103, 249-256. doi: 10.1016/S00134694(97)96160-5

Wada, J. A., and Tsuchimochi, H. (1997). Role of the claustrum in convulsive evolution of visual afferent and partial nonconvulsive seizure in primates. Epilepsia 38, 897-906. doi: 10.1111/j.1528-1157.1997.tb01255.x

Wilhite, B. L., Teyler, T. J., and Hendricks, C. (1986). Functional relations of the rodent claustral-entorhinal-hippocampal system. Brain Res. 365, 54-60. doi: 10.1016/0006-8993(86)90721-3

Wilson, S. K. (1912). Progressive lenticular degeneration: a familial nervous disease associated with cirrhosis of the liver. Brain 34, 295-507. doi: 10.1093/brain/34.4.295

Wisco, J. J., Killiany, R. J., Guttmann, C. R., Warfield, S. K., Moss, M. B., and Rosene, D. L. (2008). An MRI study of age-related white and gray matter volume changes in the rhesus monkey. Neurobiol. Aging 29, 1563-1575. doi: 10.1016/j.neurobiolaging.2007.03.022

Witter, M. P., Room, P., Groenewegen, H. J., and Lohman, A. H. (1988). Reciprocal connections of the insular and piriform claustrum with limbic cortex: an anatomical study in the cat. Neuroscience 24, 519-539. doi: 10.1016/03064522(88)90347-8

Yamamoto, R., Iseki, E., Murayama, N., Minegishi, M., Marui, W., Togo, T., et al. (2007). Correlation in Lewy pathology between the claustrum and visual areas in brains of dementia with Lewy bodies. Neurosci. Lett. 415, 219-224. doi: 10.1016/j.neulet.2007.01.029
Yamamoto, T., and Kawamura, Y. (1975). Cortical responses to electrical and gustatory stimuli in the rabbit. Brain Res. 94, 447-463. doi: 10.1016/00068993(75)90228-0

Zingg, B., Hintiryan, H., Gou, L., Song, M. Y., Bay, M., Bienkowski, M. S., et al. (2014). Neural networks of the mouse neocortex. Cell 156, 1096-1111. doi: 10.1016/j.cell.2014.02.023

Conflict of Interest Statement: The Associate Editor Mihail Bota declares that, despite being affiliated to the same institution as authors, the review process was handled objectively and no conflict of interest exists. The authors declare that the research was conducted in the absence of any commercial or financial relationships that could be construed as a potential conflict of interest.

Received: 03 July 2014; accepted: 09 October 2014; published online: 11 November 2014.

Citation: Torgerson CM and Van Horn JD (2014) A case study in connectomics: the history, mapping, and connectivity of the claustrum. Front. Neuroinform. 8:83. doi: 10.3389/fninf.2014.00083

This article was submitted to the journal Frontiers in Neuroinformatics.

Copyright (c) 2014 Torgerson and Van Horn. This is an open-access article distributed under the terms of the Creative Commons Attribution License (CC BY). The use, distribution or reproduction in other forums is permitted, provided the original author(s) or licensor are credited and that the original publication in this journal is cited, in accordance with accepted academic practice. No use, distribution or reproduction is permitted which does not comply with these terms. 